\title{
LUT
}

University

\section{Ambient backscatter communications over NOMA downlink channels}

Chen Weiyu, Ding Haiyang, Wang Shilian, Benevides da Costa Daniel, Gong Fengkui, Nardelli Pedro Henrique Juliano

This is a Final draft version of a publication

published by IEEE

in China Communications

DOI: $\quad 10.23919 / J C C .2020 .06 .007$

Copyright of the original publication: (C) 2020 IEEE

Please cite the publication as follows:

W. Chen, H. Ding, S. Wang, D. B. da Costa, F. Gong and P. H. J. Nardelli, "Ambient backscatter communications over NOMA downlink channels," in China Communications, vol. 17, no. 6, pp. 80-100, June 2020, doi: 10.23919/JCC.2020.06.007.

(C) 2020 IEEE. Personal use of this material is permitted. Permission from IEEE must be obtained for all other uses, in any current or future media, including reprinting/republishing this material for advertising or promotional purposes, creating new collective works, for resale or redistribution to servers or lists, or reuse of any copyrighted component of this work in other works.

This is a parallel published version of an original publication. This version can differ from the original published article. 


\title{
Ambient Backscatter Communications over NOMA Downlink Channels
}

\author{
Weiyu Chen, Haiyang Ding, Shilian Wang, Daniel Benevides da Costa, \\ Fengkui Gong, and Pedro Henrique Juliano Nardelli
}

\begin{abstract}
In this paper, we investigate the performance of commensal ambient backscatter communications $(\mathrm{AmBC})$ that ride on a non-orthogonal multiple access (NOMA) downlink transmission, in which a backscatter device (BD) splits part of its received signals from the base station (BS) for energy harvesting, and backscatters the remaining received signals to transmit information to a cellular user. Specifically, under the power consumption constraint at BD and the peak transmit power constraint at BS, we derive the optimal reflection coefficient at BD, the optimal total transmit power at BS, and the optimal power allocation at BS for each transmission block to maximize the ergodic capacity of the ambient backscatter transmission on the premise of preserving the outage performance of the NOMA downlink transmission. Furthermore, we consider a scenario where the BS is restricted by a maximum allowed average transmit power and the reflection coefficient at BD is fixed due to BD's low-complexity nature. An algorithm is developed to determine the optimal total transmit power and power allocation at BS for this scenario. Also, a low-complexity algorithm is proposed for this scenario to reduce the computational complexity and the signaling overheads. Finally, the performance of the derived solutions are studied and compared via numerical simulations.
\end{abstract}

W. Chen and S. Wang are with College of Electronic Science, National University of Defense Technology, Changsha 410073, China (email: chenweiyu14@nudt.edu.cn,wangs1@nudt.edu.cn). This work was supported in part by the National Key R\&D Program of China under Grant 2018YFE0100500; by the National Natural Science Foundation of China under Grant 61871387, Grant 61861041, and Grant 61871471; by the Natural Science Basic Research Program of Shaanxi under Grant 2019JM-019; and by the NUDT Research Fund under Grant ZK17-03-08.

H. Ding is with College of Information and Communication, National University of Defense Technology, Xi'an 710106, China (email: dinghy2003@ hotmail.com).

D. B. da Costa is with the Department of Computer Engineering, Federal University of Ceará, Sobral 62010-560, Brazil (email: danielbcosta@ieee.org).

F. Gong is with the State Key Laboratory of Integrated Service Networks, Xidian University, Xi'an 710071, China (e-mail: fkgong@xidian.edu.cn).

P. H. J. Nardelli is with the School of Energy Systems, Lappeenranta University of Technology, Lappeenranta 53850, Finland (e-mail: Pedro.Juliano.Nardelli@lut.fi).

Corresponding author: Shilian Wang. 


\section{Index Terms}

Power-domain NOMA, ambient backscatter communications, IoT, wireless-powered devices, optimization.

\section{INTRODUCTION}

Non-orthogonal multiple access (NOMA), whose key idea is to allow more than one user to use the same time, frequency, and code resources to access the network, has been recognized as a promising technique for the imminent fifth-generation $(5 \mathrm{G})$ era to improve spectrum efficiency [1], [2]. As one of many specific techniques of NOMA, power-domain NOMA, whose key idea is to distinguish different users by allocating different power levels to them, has attracted significant research interests due to its high compatibility with other techniques and low implementation complexity [3]. Specifically, in power-domain $\mathrm{NOMA}^{1}$, the users with a worse channel condition are allocated with a higher power level. By using the successive interference cancellation (SIC) technique [4], the users with a better channel condition can firstly decode and subtract the intended signals for the users with a worse channel condition from their observations, and then recover their own information. In this way, NOMA can achieve a 30\% system-level performance improvement over orthogonal multiple access (OMA) [5]. Some in-depth studies related to NOMA can be found in [6]-[8]. Specifically, the outage probability and the achievable sum data rate for NOMA uplink transmission were analyzed in [6], whereas the bit error rates under different channel fading types for NOMA downlink transmission were investigated in [7]. Additionally, the authors in [8] introduced a cooperation scheme, which can achieve a diversity order of $K$ at all the $K$ NOMA users.

On the other hand, ambient backscatter communication (AmBC), whose key idea is to transmit the information from a backscatter device (BD) to its corresponding receiver by backscattering the signals from an ambient radio-frequency (RF) source [9], is emerging as a potential technique for green Internet-of-Things (IoT) since it can improve spectrum efficiency and energy efficiency simultaneously [9], [10]. Specifically, in AmBC, the BD varies its load impedance to change the amplitude and/or phase of the backscattered signals. In this way, information is transmitted. A branch of $\mathrm{AmBC}$ is cooperative $\mathrm{AmBC}(\mathrm{CABC})$ [11], in which the $\mathrm{AmBC}$ receiver also recovers the information from the RF source, so that the interference from the RF source can

\footnotetext{
${ }^{1}$ This paper mainly focuses on power-domain NOMA, which we refer to as NOMA for conciseness in the rest of the paper.
} 
be eliminated at the $\mathrm{AmBC}$ receiver before it recovers the information from the $\mathrm{BD}$. In the context of CABC, the authors in [11] derived an SIC-based detector, which was adopted at the AmBC receivers in [12] and [13]. Particularly, the work in [12] proposed a scheme called Riding on the Primary (ROP), in which a primary transmitter transmits information to a primary receiver. Meanwhile, a BD (secondary transmitter) uses the primary signals as its energy source to power its circuit operation and meanwhile as the carrier to transmit information to a secondary SIC-based receiver. For different constraints, the optimal reflection coefficient at the BD and the optimal transmit power at the primary transmitter were derived to maximize the ergodic capacity at the secondary receiver. For a similar scenario, the authors in [13] further proposed three symbiotic schemes (commensal scheme, parasitic scheme, and competitive scheme) in terms of the relationship between the primary transmission and the secondary transmission, and derived the optimal transmit power at the primary transmitter for each scheme.

Both NOMA and AmBC have great potential in enhancing the spectrum efficiency. It is desirable to combine these two techniques to further boost the spectrum efficiency, since the AmBC technique can reuse the same spectrum occupied by a NOMA communication system. In this regard, the authors in [14] proposed a Backscatter-NOMA scheme, in which the AmBC is conducted between a $\mathrm{BD}$ and one of the two cellular users by riding on the NOMA downlink signals. The analytical expressions of the outage probabilities and the ergodic rates for both the NOMA downlink transmission and the backscatter transmission were developed under given system parameters setups. However, up to now, it is still not clear how to adaptively adjust the system parameters to ensure the quality-of-service (QoS) of NOMA downlink transmission and meanwhile to maximize the throughput of $A m B C$, which motivates our work. In addition, it is desirable that all the energy consumption for $\mathrm{AmBC}$ at the BD is supplied by the NOMA downlink signals to further improve the energy efficiency, which has not been considered yet in the context of NOMA with AmBC. This work aims to address these problems, and the main contributions can be summarized as follows:

i) Focusing on a two-user NOMA downlink scenario where a BD splits part of its received signals from the base station (BS) for energy harvesting and backscatters the remaining received signals to transmit information to the cell-center user, we maximize the ergodic capacity of the backscatter transmission on the premise of preserving the outage performance of the NOMA downlink transmission. Specifically, under the power consumption constraint at BD and the peak transmit power constraint at BS, the theoretical expressions of the optimal reflection coefficient 


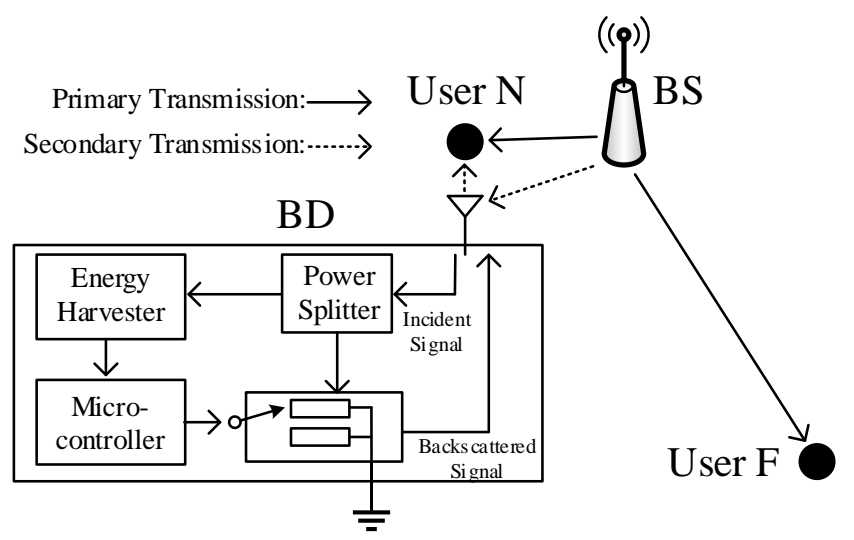

Fig. 1: System model.

at $\mathrm{BD}$, the optimal total transmit power at $\mathrm{BS}$, and the optimal power allocation at $\mathrm{BS}$ are derived for each transmission block.

ii) We further consider a scenario where the BS is restricted by a maximum allowed average transmit power and the reflection coefficient at BD is fixed due to BD's low-complexity nature. For this scenario, by converting the original problem into a convex one, an algorithm is developed to determine the optimal total transmit power and the optimal power allocation at BS for each transmission block under a given fixed reflection coefficient. Furthermore, a low-complexity algorithm is proposed to reduce the computational complexity and the signaling overheads, whose performance loss is shown to be not significant compared with the optimal solutions.

The rest of the paper is organized as follows. Section II illustrates the system model, introduces all the involved constraints, and formulates the problem. The solutions for both scenarios are derived in Section III. Section IV provides representative numerical results and then makes a comprehensive discussion. Finally, Section V concludes the paper.

\section{System Model AND PROBlem Formulation}

\section{A. System Model}

As shown in Fig. 1, we consider a commensal ambient backscatter transmission that rides on a NOMA downlink transmission ${ }^{2}$, which we refer to as secondary transmission (ST) and primary

\footnotetext{
${ }^{2}$ In practice, the performance of the backscatter transmission is vulnerable to unexpected interference from other communication systems due to its passive nature. This problem may become even more involved as a result of emerging heterogeneous protocols [15] and different requirements of bandwidth [16], which will be addressed in future works.
} 
transmission (PT), respectively. Specifically, the block fading channel model is considered [12], where the channel coefficients remain unchanged within each transmission block (a.k.a. fading block) $n$, but may vary for different blocks. In the PT, a BS superposes and broadcasts the information of a cell-center user (denoted by $\mathrm{N}$ ) and that of a cell-edge user (denoted by F) over the same spectrum, but with different transmit powers, $P_{\mathrm{N}}(n)$ and $P_{\mathrm{F}}(n)$, respectively. The PT works in a delay-constrained transmission mode [17] and the target rates at user $\mathrm{N}$ and user $\mathrm{F}$ are $R_{\mathrm{N}}$ and $R_{\mathrm{F}}$, respectively. In the $\mathrm{ST}$, a BD splits its received signals from the BS into two parts. One part is modified and backscattered to user $\mathrm{N}$ for information transmission, while another part is used for energy harvesting to power the circuit operation at BD. For convenience, we refer to the percentage of the power split to conduct the backscatter transmission as the reflection coefficient and denote it by $\rho(n)$, where $0 \leq \rho(n) \leq 1$.

Let $h_{\mathrm{BN}}, h_{\mathrm{BF}}, h_{\mathrm{BD}}$, and $h_{\mathrm{DN}}$ represent the channel coefficients pertaining to the BS-N, BS-F, $\mathrm{BS}-\mathrm{BD}$, and BD-N links, respectively. The received signal at user $\mathrm{N}$ during the $n$-th block can be written as ${ }^{34}$

$$
\begin{aligned}
y_{\mathrm{N}}(n)= & \left(\sqrt{P_{\mathrm{N}}(n)} x_{\mathrm{N}}(n)+\sqrt{P_{\mathrm{F}}(n)} x_{\mathrm{F}}(n)\right) h_{\mathrm{BN}}(n)+n_{\mathrm{N}}(n) \\
& +\sqrt{\rho(n) \eta_{\mathrm{B}}}\left(\sqrt{P_{\mathrm{N}}(n)} x_{\mathrm{N}}(n)+\sqrt{P_{\mathrm{F}}(n)} x_{\mathrm{F}}(n)\right) x_{\mathrm{D}}(n) h_{\mathrm{BD}}(n) h_{\mathrm{DN}}(n),
\end{aligned}
$$

where $x_{\mathrm{N}}(n), x_{\mathrm{F}}(n)$, and $x_{\mathrm{D}}(n)$ denote the normalized intended signals from $\mathrm{BS}$ to user $\mathrm{N}$, to user $\mathrm{F}$, and the normalized intended signal from BD to user $\mathrm{N}$, respectively $\left(E\left\{\left|x_{\mathrm{N}}(n)\right|^{2}\right\}=\right.$ $\left.E\left\{\left|x_{\mathrm{F}}(n)\right|^{2}\right\}=E\left\{\left|x_{\mathrm{D}}(n)\right|^{2}\right\}=1\right), n_{\mathrm{N}}(n)$ represents the zero-mean additive white Gaussian noise (AWGN) at user $\mathrm{N}$ with variance $\sigma_{\mathrm{N}}^{2}$, and $0<\eta_{\mathrm{B}} \leq 1$ is the backscatter efficiency at $\mathrm{BD}^{5}$. On the other hand, the received signal at user $\mathrm{F}$ during the $n$-th block can be represented as 6

$$
y_{\mathrm{F}}(n)=\left(\sqrt{P_{\mathrm{N}}(n)} x_{\mathrm{N}}(n)+\sqrt{P_{\mathrm{F}}(n)} x_{\mathrm{F}}(n)\right) h_{\mathrm{BF}}(n)+n_{\mathrm{F}}(n),
$$

\footnotetext{
${ }^{3}$ Strictly speaking, the arrival of the backscattered signals is later than that of the signals from the BS. In this paper, we assume that this delay is negligible as in [12]. This assumption is reasonable since the BD is typically located close to the user.

${ }^{4}$ A perfect symbol-level synchronization between the PT and the ST is assumed [12]. Otherwise, the backscattered signal may be distorted when it passes the matched filter at user $\mathrm{N}$ due to the spectrum growth phenomenon [18], which will be investigated in future works.

${ }^{5}$ The reflection coefficient denotes the tradeoff between energy harvesting and backscatter transmission, whereas the backscatter efficiency depicts the implementation efficiency of backscatter operation. In general, the backscatter efficiency is less than one due to the imperfectness of the hardware circuit at the BD.

${ }^{6}$ The interference from $\mathrm{BD}$ to user $\mathrm{F}$ is assumed to be negligible as in [13], which is reasonable due to the double fading effect.
} 
where $n_{\mathrm{F}}(n)$ represents the AWGN at user $\mathrm{F}$ with variance $\sigma_{\mathrm{F}}^{2}$.

For conciseness, we omit the block index $n$ hereafter. Using the SIC technique, user $\mathrm{N}$ first decodes and subtracts $x_{\mathrm{F}}$ from its observations. Afterwards, user $\mathrm{N}$ decodes and subtracts $x_{\mathrm{N}}$ from its observations. Finally, user $\mathrm{N}$ decodes $x_{\mathrm{D}}{ }^{7}$. The corresponding signal-to-interferenceplus-noise ratio (SINR) at user $\mathrm{N}$ to decode $x_{\mathrm{F}}$ can be given by

$$
\gamma_{\mathrm{NDF}}=\frac{P_{\mathrm{F}}\left|h_{\mathrm{BN}}\right|^{2}}{P_{\mathrm{N}}\left|h_{\mathrm{BN}}\right|^{2}+\rho \eta_{\mathrm{B}} P_{\mathrm{B}}\left|h_{\mathrm{BD}} h_{\mathrm{DN}}\right|^{2}+\sigma_{\mathrm{N}}^{2}},
$$

where $P_{\mathrm{B}} \triangleq P_{\mathrm{N}}+P_{\mathrm{F}}$ denotes the total transmit power at BS. Provided that user $\mathrm{N}$ decodes $x_{\mathrm{F}}$ successfully, the SINR at user $\mathrm{N}$ to decode $x_{\mathrm{N}}$ can be written as

$$
\gamma_{\mathrm{NDN}}=\frac{P_{\mathrm{N}}\left|h_{\mathrm{BN}}\right|^{2}}{\rho \eta_{\mathrm{B}} P_{\mathrm{B}}\left|h_{\mathrm{BD}} h_{\mathrm{DN}}\right|^{2}+\sigma_{\mathrm{N}}^{2}} .
$$

Conditioned on decoding both $x_{\mathrm{F}}$ and $x_{\mathrm{N}}$ successfully, the signal-to-noise ratio (SNR) at user $\mathrm{N}$ to decode $x_{\mathrm{D}}$ can be determined by

$$
\gamma_{\mathrm{NDD}}=\frac{\rho \eta_{\mathrm{B}} P_{\mathrm{B}}\left|h_{\mathrm{BD}} h_{\mathrm{DN}}\right|^{2}}{\sigma_{\mathrm{N}}^{2}} .
$$

Note that for ST, its ergodic capacity can be written as $E\left[\log _{2}\left(1+\gamma_{\mathrm{NDD}}\right)\right]$. On the other hand, user F only needs to decode $x_{\mathrm{F}}$, and the corresponding SINR can be given by

$$
\gamma_{\mathrm{FDF}}=\frac{P_{\mathrm{F}}\left|h_{\mathrm{BF}}\right|^{2}}{P_{\mathrm{N}}\left|h_{\mathrm{BF}}\right|^{2}+\sigma_{\mathrm{F}}^{2}} .
$$

\section{B. Problem Formulation}

Our goal is to maximize the ergodic capacity of ST and meanwhile to ensure that the interference from BD shall not impair the outage performance of PT. Specifically, for PT, information outage ( $\mathrm{IO}$ ) occurs when either of the target rate of user $\mathrm{N}$ (i.e., $R_{\mathrm{N}}$ ) or that of user F (i.e., $R_{\mathrm{F}}$ ) cannot be achieved. For each fading block, if IO can be avoided via a proper transmit power setup at BS when BD does not exist, the developed solutions in the following should ensure that the interference from BD does not result in IO. On the contrary, if IO definitely happens regardless of the transmit power setup at BS and the strength of the interference from $\mathrm{BD}$, the total transmit power at $\mathrm{BS}$ (i.e., $P_{\mathrm{B}}$ ) will be set to zero to save energy as in [12].

\footnotetext{
${ }^{7}$ A stronger signal is decoded ahead of a weaker signal as per the SIC rules. According to the principles of NOMA, the cell-edge user $\mathrm{F}$ is allocated with a higher power level since its channel condition is worse than that of the cell-center user $\mathrm{N}$. In addition, due to the double fading effect, the signal from BD to user $\mathrm{N}$ is much weaker than the signal from $\mathrm{BS}$ to user $\mathrm{N}$.
} 
According to the principles of NOMA, to avoid IO at user $\mathrm{N}$, it is required that

$$
\log _{2}\left(1+\gamma_{\mathrm{NDF}}\right) \geq R_{\mathrm{F}}
$$

and

$$
\log _{2}\left(1+\gamma_{\mathrm{NDN}}\right) \geq R_{\mathrm{N}}
$$

On the other hand, to avoid IO at user F, it is required that

$$
\log _{2}\left(1+\gamma_{\mathrm{FDF}}\right) \geq R_{\mathrm{F}}
$$

Apart from (7) (9), two basic constraints need to be considered. Firstly, for each fading block, if BD conducts backscatter transmission $(\rho>0)$, its harvested energy should be not less than its consumed energy. Herein we adopt a practical energy consumption model as in [12], in which the consumed energy consists of a static energy consumption and a dynamic energy consumption. The latter is proportional to the transmission rate of the ST (i.e., $\left.\log _{2}\left(1+\gamma_{\mathrm{NDD}}\right)\right)$, since a higher transmission rate requires a higher switching frequency on the switches at BD. As thus, the power consumption constraint can be represented as ${ }^{8}$

$$
(1-\rho) \eta_{\mathrm{C}} P_{\mathrm{B}}\left|h_{\mathrm{BD}}\right|^{2} \geq \epsilon_{s}+\epsilon_{d} \log _{2}\left(1+\gamma_{\mathrm{NDD}}\right),
$$

where $\eta_{\mathrm{C}}$ denotes the energy conversion efficiency at $\mathrm{BD}, \epsilon_{s}$ represents the static power consumption, and $\epsilon_{d}$ denotes the dynamic power consumption coefficient due to the switching operation. The second basic constraint is that the $\mathrm{BS}$ is restricted by a maximum allowed peak transmit power, which we denote by $P_{\text {peak }}$. The peak transmit power constraint can be written as

$$
0 \leq P_{\mathrm{B}} \leq P_{\text {peak }}
$$

Furthermore, apart from the peak transmit power constraint, the BS may also be restricted by a maximum allowed average transmit power, which we denote by $P_{\mathrm{av}}$. The average transmit power constraint can be represented as

$$
E\left\{P_{\mathrm{B}}\right\} \leq P_{\mathrm{av}}
$$

Note that when $P_{\text {peak }}<P_{\text {av }}$, the average transmit power constraint becomes trivial since it is already satisfied by the peak transmit power constraint. Therefore, we assume $P_{\text {peak }} \geq P_{\text {av }}$ in the rest of the paper, which is in line with reality.

\footnotetext{
${ }^{8}$ The harvested energy from noise is negligible since it is much lower than that from the received signals [12].
} 
Finally, we consider a fixed reflection coefficient constraint at the BD, since a continuously adjustable reflection coefficient setup involves a high implementation cost. Note that the work in [12] also considered a fixed reflection coefficient, where a one-dimension search of the optimal fixed reflection coefficient at BD was performed to maximize the ergodic capacity of the backscatter transmission. However, this one-dimension search indeed arrives at a Pareto optimal solution when considering both the ergodic capacity at the secondary receiver and the outage probability at the primary receiver, since a higher fixed reflection coefficient at BD leads to a higher outage probability at the primary receiver. Similarly, in this paper, if the reflection coefficient at BD is fixed, to preserve the outage performance of PT, the optimal value of the fixed reflection coefficient is indeed zero, which leads to a zero ergodic capacity of ST. Therefore, herein we assume that the BD can switch between a fixed reflection coefficient $\bar{\rho}$ and a zero reflection coefficient such that by properly designing the switching rules, the choice of $\bar{\rho}$ will not impair the outage performance of PT.

By summarizing the foregoing constraints, the problem can be formulated as

$$
\begin{aligned}
& \text { P1 : } \max _{\alpha(n), P_{\mathrm{B}}(n), \rho(n), \bar{\rho}} E\left\{\log _{2}\left(1+\gamma_{\mathrm{NDD}}(n)\right)\right\}, \\
& \text { s. t. } \rho(n)=0 \text { or } \rho(n)=\bar{\rho}, \\
& 0<\alpha(n)<1,0<\bar{\rho}<1,(12), \\
& \text { (7), (8), (9), and (11) for } n \in\{n \mid \rho(n)=0\}, \\
& \text { (7), (8), (9), (10), and (11) for } n \in\{n \mid \rho(n)=\bar{\rho}\},
\end{aligned}
$$

where $\alpha(n)$ denotes the percentage of the transmit power allocated to user $\mathrm{N}$ at BS within the $n$-th fading block (i.e., $\alpha(n) P_{\mathrm{B}}(n)=P_{\mathrm{N}}(n)$ ), which we refer to as power allocation (PA) factor in the rest of the paper for convenience.

\section{ERGODIC CAPACITY MAXIMIZATION}

\section{A. A Benchmark Scenario}

Before addressing P1, we first consider a benchmark scenario, where there is no average transmit power constraint and no fixed reflection coefficient constraint ${ }^{9}$. As a result, the remaining

\footnotetext{
${ }^{9}$ For the benchmark scenario considered herein, we are able to obtain simpler algorithm and solutions with a lower computational complexity. Through the comparison made in Section IV, we can find out what are the impacts of the average transmit power constraint and fixed reflection coefficient constraint.
} 
constraints are all instantaneous constraints, which means that maximizing the ergodic capacity is equivalent to maximizing the instantaneous capacity. As thus, the problem can be formulated as

$$
\begin{gathered}
\mathrm{P} 2: \max _{\alpha, P_{\mathrm{B}}, \rho} \log _{2}\left(1+\frac{\rho \eta_{\mathrm{B}} P_{\mathrm{B}}\left|h_{\mathrm{BD}} h_{\mathrm{DN}}\right|^{2}}{\sigma_{\mathrm{N}}^{2}}\right), \\
\text { s. t. } 0<\alpha<1,0 \leq \rho<1 \\
(7),(8),(9),(10),(11) .
\end{gathered}
$$

Unfortunately, P2 is not a convex problem since its objective function is not concave, which can be checked by calculating the determinant of the Hessian matrix of the objective function. Another difficulty in solving P2 is that the three optimization variables are tightly coupled in constraints (7) and (8). To address P2, we first investigate its feasibility for a given fading block as follows.

Proposition 1: If $P_{\text {peak }}<P_{\mathrm{LB}} \triangleq \max \left\{\frac{\left(2^{\left.R_{\mathrm{F}} 2^{R_{\mathrm{N}}}-1\right) \sigma_{\mathrm{N}}^{2}}\right.}{\left|h_{\mathrm{BN}}\right|^{2}}, \frac{\left(2^{R_{\mathrm{F}}}-1\right) \sigma_{\mathrm{F}}^{2}}{\left|h_{\mathrm{BF}}\right|^{2}}+\frac{2^{R_{\mathrm{F}}}\left(2^{R_{\mathrm{N}}}-1\right) \sigma_{\mathrm{N}}^{2}}{\left|h_{\mathrm{BN}}\right|^{2}}\right\}$, constraints (7) (9) and (11) cannot be satisfied simultaneously and thus P2 is infeasible. On the other hand, if $\eta_{\mathrm{C}} P_{\text {peak }}\left|h_{\mathrm{BD}}\right|^{2}<\epsilon_{s}$, constraints (10) and (11) cannot be satisfied simultaneously and thus P2 is infeasible. On the contrary, if neither of the two inequalities above is satisfied, P2 is feasible.

Proof: Please refer to Appendix A-1.

Remark 1: Note that we cope with the two cases that lead to infeasibility in different ways. When $P_{\text {peak }}<P_{\mathrm{LB}}, \mathrm{P} 2$ is infeasible because IO definitely happens for PT regardless of the transmit power setup at $\mathrm{BS}$ and the strength of the interference from BD. In this case, $P_{\mathrm{B}}$ is set to zero to save energy and thus the instantaneous capacity of ST is zero. In comparison, when $\eta_{\mathrm{C}} P_{\text {peak }}\left|h_{\mathrm{BD}}\right|^{2}<\epsilon_{s}$, P2 is infeasible because the harvested energy at BD cannot support the backscatter transmission regardless of the reflection coefficient setup at BD and the total transmit power setup at BS. In this case, the BD can only conduct energy harvesting (i.e., $\rho=0$ ) and thus the instantaneous capacity of ST is also zero, but IO does not necessarily happen for PT.

Corollary 1: For both $\mathrm{P} 1$ and $\mathrm{P} 2$, the outage probability of PT can be written as $P_{\text {out }}=$ $\operatorname{Pr}\left\{P_{\text {peak }}<P_{\mathrm{LB}}\right\}$.

Proof: Please refer to Appendix A-1.

Next, based on Proposition 1, we provide the solutions to P2 for feasible fading blocks as follows. 
Proposition 2: For a given fading block, provided that P2 is feasible, given the PA factor and the total transmit power at $\mathrm{BS}$, the optimal reflection coefficient at $\mathrm{BD}$ for $\mathrm{P} 2$ can be given by

$$
\rho^{*}=\min \left\{\hat{\rho}, \frac{\left|h_{\mathrm{BN}}\right|^{2} \frac{\alpha P_{\mathrm{B}}}{\left(2^{R_{\mathrm{N}}}-1\right)}-\sigma_{\mathrm{N}}^{2}}{\eta_{\mathrm{B}} P_{\mathrm{B}}\left|h_{\mathrm{BD}} h_{\mathrm{DN}}\right|^{2}}, \frac{\left|h_{\mathrm{BN}}\right|^{2} \frac{\left(1-\alpha 2^{R_{F}}\right) P_{\mathrm{B}}}{\left(2^{R_{\mathrm{F}}}-1\right)}-\sigma_{\mathrm{N}}^{2}}{\eta_{\mathrm{B}} P_{\mathrm{B}}\left|h_{\mathrm{BD}} h_{\mathrm{DN}}\right|^{2}}\right\},
$$

where $\hat{\rho}$ is the unique solution of the equation $(1-\rho) \eta_{\mathrm{C}} P_{\mathrm{B}}\left|h_{\mathrm{BD}}\right|^{2}=\epsilon_{s}+\epsilon_{d} \log _{2}\left(1+\gamma_{\mathrm{NDD}}\right)$. Furthermore, with the reflection coefficient equal to $\rho^{*}$ and given the total transmit power at BS, the optimal PA factor at BS for P2 can be written as

$$
\alpha^{*}= \begin{cases}\alpha_{\mathrm{U}}, & \hat{\alpha}>\alpha_{\mathrm{U}} \\ \hat{\alpha}, & \alpha_{\mathrm{L}} \leq \hat{\alpha} \leq \alpha_{\mathrm{U}} \\ \alpha_{\mathrm{L}}, & \hat{\alpha}<\alpha_{\mathrm{L}},\end{cases}
$$

where $\alpha_{\mathrm{U}} \triangleq \min \left\{\frac{1}{2^{R_{\mathrm{F}}}}\left(1-\frac{\left(2^{R_{\mathrm{F}}}-1\right) \sigma_{\mathrm{N}}^{2}}{P_{\mathrm{B}}\left|h_{\mathrm{BN}}\right|^{2}}\right), \frac{1}{2^{R_{\mathrm{F}}}}\left(1-\frac{\left(2^{R_{\mathrm{F}}}-1\right) \sigma_{\mathrm{F}}^{2}}{P_{\mathrm{B}}\left|h_{\mathrm{BF}}\right|^{2}}\right)\right\}, \hat{\alpha} \triangleq \frac{2^{R_{\mathrm{N}}-1}}{2^{R_{\mathrm{F}} 2^{R_{\mathrm{N}}}-1}}$, and $\alpha_{\mathrm{L}} \triangleq$ $\frac{\left(2^{R_{\mathrm{N}}}-1\right) \sigma_{\mathrm{N}}^{2}}{P_{\mathrm{B}}\left|h_{\mathrm{BN}}\right|^{2}}$. Finally, with the reflection coefficient equal to $\rho^{*}$ and the PA factor equal to $\alpha^{*}$, the optimal total transmit power at BS for $\mathrm{P} 2$ is $P_{\mathrm{B}}^{*}=P_{\text {peak }}$.

Proof: Please refer to Appendix A-2.

Remark 2: It follows from Proposition 2 that the reflection coefficient at BD is restricted by two factors. Firstly, it cannot be too large because the dynamic power consumption at the BD (i.e., $\left.\epsilon_{d} \log _{2}\left(1+\gamma_{\mathrm{NDD}}\right)\right)$ increases with the reflection coefficient, whereas the harvested energy (i.e., $\left.(1-\rho) \eta_{\mathrm{C}} P_{\mathrm{B}}\left|h_{\mathrm{BD}}\right|^{2}\right)$ decreases with the reflection coefficient. Secondly, it cannot be too large so that the interference from the BD will not lead to information outage at user N. Meanwhile, it is shown that the reflection coefficient should be continuously adjustable to achieve the optimal performance, which requires a complex circuit at the $\mathrm{BD}$. On the other hand, to boost the performance of the ST, it follows that the optimal total transmit power at BS is always the peak transmit power. This is intuitive since the transmission rate is a monotonically increasing function of the transmit power, according to (5). However, in practice, the BS may be restricted by a maximum allowed average transmit power, which may be lower than the peak transmit power. In what follows, we go back to P1 to investigate the scenario in which the BS is restricted by a maximum allowed average transmit power and the reflection coefficient is fixed. 


\section{B. Solutions to P1}

For P1, since constraint (12) is not an instantaneous constraint, we have to optimize the ergodic capacity of ST over $N$ successive fading blocks. In the following, due to the complexity of the problem, assuming a given value of $\bar{\rho}$, we derive the optimal PA factor, the optimal total transmit power, as well as the switching rules of the reflection coefficient for each fading block, whereas the optimal value of $\bar{\rho}$ can be determined by conducting a one-dimension search of over the space $(0,1)$. Before that, considering the instantaneous constraints $(7) \sim(11)$, we investigate the feasibility for a single fading block and present the switching rules of the reflection coefficient as follows.

Let $\mathcal{F}_{0}$ denote the set of the indexes of the fading blocks in which IO definitely happens for PT regardless of the transmit power setup at BS and the strength of the interference from BD. For these blocks, even if the reflection coefficient $\rho$ is switched to zero, constraints (7) (9) and (11) cannot be satisfied simultaneously such that $P_{\mathrm{B}}$ is set to zero to save energy as before (i.e., $\forall n \in \mathcal{F}_{0}, P_{\mathrm{B}}^{*}(n)=0$ ). Let $\mathcal{F}_{1}$ represent the set of the indexes of the fading blocks in which IO would definitely happen if the reflection coefficient is switched to the fixed value $\bar{\rho}$, but can be avoided by switching the reflection coefficient to zero and adopting a proper transmit power setup at BS. For these blocks, constraints (7) (11) cannot be satisfied simultaneously with reflection coefficient equal to $\bar{\rho}$, such that $\rho$ is switched to zero to ensure the outage performance of PT. Meanwhile, the total transmit power at BS is set to its minimum required value to avoid IO in these blocks. Applying the derived results in Appendix A-1, we have $P_{\mathrm{B}}^{*}(n)=P_{\mathrm{LB}}(n)$ and $\alpha^{*}(n)=\alpha_{\mathrm{LB}}(n)$ for all $n \in \mathcal{F}_{1}$, where $\alpha_{\mathrm{LB}}(n)$ is given by (A.11). Note that determining $P_{\mathrm{B}}^{*}(n)$ for $n \in \mathcal{F}_{0} \cup \mathcal{F}_{1}$ is useful when we consider the average transmit power constraint afterwards. Finally, let $\mathcal{F}_{2}$ denote the set of the indexes of the fading blocks in which IO can be avoided even if the reflection coefficient is switched to the fixed value $\bar{\rho}$. For these blocks, by properly setting the transmit power at BS, all of the instantaneous constraints can be satisfied simultaneously with the reflection coefficient equal to $\bar{\rho}$, such that the reflection coefficient is switched to $\bar{\rho}$ to conduct the backscatter transmission. Next, for conciseness, we

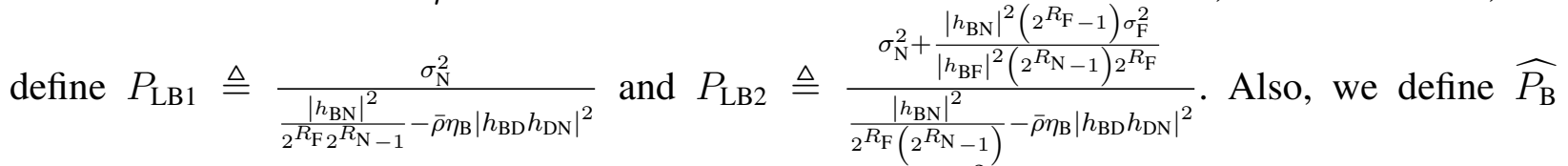
as the unique solution of the equation $(1-\bar{\rho}) \eta_{\mathrm{C}} P_{\mathrm{B}}\left|h_{\mathrm{BD}}\right|^{2}=\epsilon_{s}+\epsilon_{d} \log _{2}\left(1+\bar{\gamma}_{\mathrm{NDD}}\right)$, where $\bar{\gamma}_{\mathrm{NDD}} \triangleq \frac{\bar{\rho} \eta_{\mathrm{B}} P_{\mathrm{B}}\left|h_{\mathrm{BD}} h_{\mathrm{DN}}\right|^{2}}{\sigma_{\mathrm{N}}^{2}}$. Based on these definitions, the belonging of a given fading block can be 
determined by the following Proposition.

Proposition 3: For the $n$-th block, it follows that $n \in \mathcal{F}_{0}$ if and only if $P_{\text {peak }}<P_{\mathrm{LB}}(n)$. Otherwise, if the inequalities $P_{\text {peak }}<P_{\mathrm{M}}(n) \triangleq \max \left\{\widehat{P_{\mathrm{B}}}(n), P_{\mathrm{LB} 1}(n), P_{\mathrm{LB} 2}(n)\right\}$ or $\frac{\left|h_{\mathrm{BN}}(n)\right|^{2}}{\left(2^{\left.R_{\mathrm{F}} 2^{R_{\mathrm{N}}}-1\right)} \leq\right.}$ $\bar{\rho} \eta_{\mathrm{B}}\left|h_{\mathrm{BD}}(n) h_{\mathrm{DN}}(n)\right|^{2}$ holds, we have $n \in \mathcal{F}_{1}$. In other cases, it follows that $n \in \mathcal{F}_{2}$.

Proof: Please refer to Appendix B-1.

Since the optimal transmit power setup has been determined for $n \in \mathcal{F}_{0} \cup \mathcal{F}_{1}$, we only need to determine the optimal total transmit power $P_{\mathrm{B}}^{*}(n)$ and the optimal PA factor $\alpha^{*}(n)$ for $n \in \mathcal{F}_{2}$. As thus, the problem can be formulated as

$$
\text { P1a : } \max _{\alpha(n), P_{\mathrm{B}}(n), n \in \mathcal{F}_{2}} \frac{1}{N} \sum_{\substack{n=1 \\ n \in \mathcal{F}_{2}}}^{N} \log _{2}\left(1+\frac{\bar{\rho} \eta_{\mathrm{B}} P_{\mathrm{B}}(n)\left|h_{\mathrm{BD}}(n) h_{\mathrm{DN}}(n)\right|^{2}}{\sigma_{\mathrm{N}}^{2}}\right)
$$$$
\text { s. t. (7), (8), (9), (10), (11), (12), where } \rho=\bar{\rho} \text {. }
$$

Unfortunately, P1a is not a convex problem since constraint (7) is not a convex constraint, which can be validated by calculating the first-order leading principal minor of the Hessian matrix of $\left(R_{\mathrm{F}}-\log _{2}\left(1+\gamma_{\mathrm{NDF}}\right)\right)$ in constraint (7). Another difficulty in solving P1a is that the solutions of each fading blocks are coupled by the objective function and constraint (12). To address these difficulties, we first present the optimal PA factor for P1a as follows.

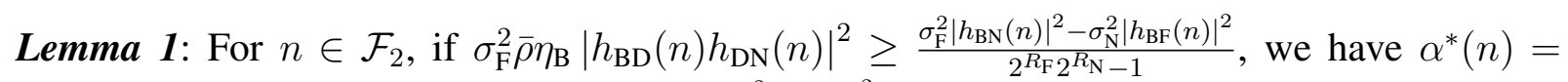

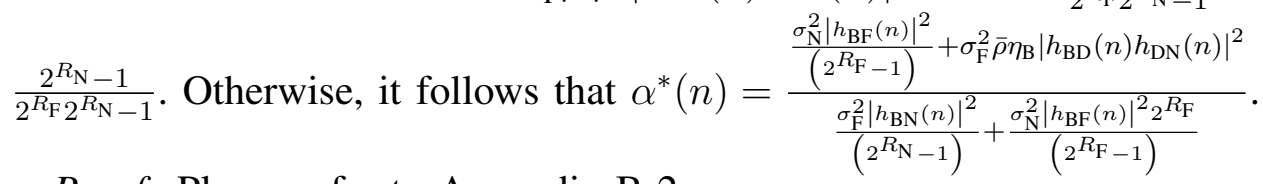

Proof: Please refer to Appendix B-2.

Next, by adopting the derived optimal PA factor $\alpha^{*}(n)$ given in Lemma 1 and replacing constraints $(7) \sim(10)$ with their equivalent constraints $($ B.1) $\sim(B .4)$, P1a can be reformulated as the following convex problem ${ }^{10}$.

$$
\begin{aligned}
\text { P1b : } \max _{P_{\mathrm{B}}(n), n \in \mathcal{F}_{2}} \frac{1}{N} \sum_{\substack{n=1 \\
n \in \mathcal{F}_{2}}}^{N} \log _{2}\left(1+\frac{\bar{\rho} \eta_{\mathrm{B}} P_{\mathrm{B}}(n)\left|h_{\mathrm{BD}}(n) h_{\mathrm{DN}}(n)\right|^{2}}{\sigma_{\mathrm{N}}^{2}}\right), \\
\text { s. t. } P_{\mathrm{M}}(n) \leq P_{\mathrm{B}}(n) \leq P_{\text {peak }}, n \in \mathcal{F}_{2}, \\
\\
\frac{1}{N} \sum_{n=1}^{N} P_{\mathrm{B}}(n) \leq P_{\mathrm{av}} .
\end{aligned}
$$

\footnotetext{
${ }^{10}$ Note that (B.1) definitely holds for $n \in \mathcal{F}_{2}$, and (B.2) definitely holds when $\alpha(n)=\alpha^{*}(n)$. Therefore, these two constraints are not directly displayed in P1b.
} 
Note that if $\sum_{\substack{n=1 \\ n \in \mathcal{F}_{1}}}^{N} P_{\mathrm{B}}^{*}(n)+\sum_{\substack{n=1 \\ n \in \mathcal{F}_{2}}}^{N} P_{\mathrm{M}}(n)>N P_{\mathrm{av}}$, P1b is infeasible. On the other hand, if constraint $\sum_{n=1}^{N} P_{\mathrm{B}}(n) \leq N P_{\mathrm{av}}$ can be satisfied when we adopt $P_{\mathrm{B}}^{*}(n)=P_{\text {peak }}$ for all $n \in \mathcal{F}_{2}$, the solution to $\mathrm{P} 1 \mathrm{~b}$ is that $\forall n \in \mathcal{F}_{2}, P_{\mathrm{B}}^{*}(n)=P_{\text {peak. }}$. In the following, we consider the case in which P1b is feasible, and there exists at least an $\hat{n} \in \mathcal{F}_{2}$ subject to $P_{\mathrm{B}}^{*}(\hat{n})<P_{\text {peak }}$.

Proposition 4: Provided that there exists at least an $\hat{n} \in \mathcal{F}_{2}$ subject to $P_{\mathrm{B}}^{*}(\hat{n})<P_{\text {peak }}$, for $n \in \mathcal{F}_{2}$, the optimal total transmit power at BS can be given by

$$
P_{\mathrm{B}}^{*}(n)= \begin{cases}P_{\mathrm{M}}(n), & \frac{\lambda_{\mathrm{av}}^{*} \ln 2}{f(n)}>\frac{1}{1+f(n) P_{\mathrm{M}}(n)}, \\ \frac{1}{\lambda_{\mathrm{av}}^{*} \ln 2}-\frac{1}{f(n)}, & \frac{1}{1+f(n) P_{\text {peak }}} \leq \frac{\lambda_{\mathrm{av}}^{*} \ln 2}{f(n)} \leq \frac{1}{1+f(n) P_{\mathrm{M}}(n)}, \\ P_{\text {peak }}, & 0 \leq \frac{\lambda_{\mathrm{av}}^{*} \ln 2}{f(n)}<\frac{1}{1+f(n) P_{\text {peak }}},\end{cases}
$$

where $f(n) \triangleq \frac{\bar{\rho} \eta_{\mathrm{B}}\left|h_{\mathrm{BD}}(n) h_{\mathrm{DN}}(n)\right|^{2}}{\sigma_{\mathrm{N}}^{2}}$, and $\lambda_{\mathrm{av}}^{*}$ can be determined by solving $\frac{1}{N} \sum_{n=1}^{N} P_{\mathrm{B}}^{*}(n)=P_{\mathrm{av}}$.

Proof: Please refer to Appendix B-3.

Remark 3: Note that $\lambda_{\mathrm{av}}^{*}$ can be regarded as a parameter to adjust the transmit power lever at the BS. Specifically, for all $n \in \mathcal{F}_{2}$, the total transmit power should be within $\left[P_{\mathrm{M}}(n), P_{\text {peak }}\right]$. When $\lambda_{\mathrm{av}}^{*}$ becomes larger, the optimal total transmit power approaches to or equals $P_{\mathrm{M}}(n)$. On the contrary, when $\lambda_{\mathrm{av}}^{*}$ becomes smaller, the optimal total transmit power approaches to or equals $P_{\text {peak }}$. This observation is useful when we need to adjust $\lambda_{\mathrm{av}}^{*}$ to satisfy the average transmit power constraint, $\frac{1}{N} \sum_{n=1}^{N} P_{\mathrm{B}}^{*}(n)=P_{\mathrm{av}}$.

So far, we have arrived at the optimal PA factor, the optimal total transmit power, as well as the switching rules of the reflection coefficient for each fading block with a given value of $\bar{\rho}$, which are summarized in Table I. Also, the procedures to determine these solutions for a given fading block is illustrated in Fig. 2 provided that constraint $\frac{1}{N} \sum_{n=1}^{N} P_{\mathrm{B}}(n) \leq P_{\mathrm{av}}$ cannot be satisfied when we adopt $P_{\mathrm{B}}^{*}(n)=P_{\text {peak }}$ for all $n \in \mathcal{F}_{2}$ (otherwise, $\forall n \in \mathcal{F}_{2}, P_{\mathrm{B}}^{*}(n)=P_{\text {peak }}$ ). As can be observed from Fig. 2, the developed algorithm for P1 has a high computational complexity due to the alternation between adjusting $\lambda_{\mathrm{av}}^{*}$ and calculating the optimal transmit power setup, although it can achieve the optimal performance. On the other hand, the developed algorithm requires the channel state information (CSI) of the BS-BD link and that of the BD-N link, which may lead to heavy signaling overheads at the BS. To address these problems, we further propose a low-complexity algorithm as follows. 
TABLE I: Solutions to P1.

\begin{tabular}{|c|c|c|}
\hline \multicolumn{2}{|r|}{$\begin{array}{c}\text { Types of the fading block } \\
\text { (cf. Proposition 3) }\end{array}$} & Solutions \\
\hline \multicolumn{2}{|r|}{$n \in \mathcal{F}_{0}$} & $\begin{array}{l}\rho^{*}(n)=0, \\
P_{\mathrm{B}}^{*}(n)=0\end{array}$ \\
\hline \multicolumn{2}{|r|}{$n \in \mathcal{F}_{1}$} & $\begin{array}{c}\rho^{*}(n)=0, \\
\alpha^{*}(n)=\alpha_{\mathrm{LB}}(n), \text { cf. }(\mathrm{A} .11), \\
P_{\mathrm{B}}^{*}(n)=P_{\mathrm{LB}}(n), \text { cf. Proposition } 1\end{array}$ \\
\hline \multirow[t]{2}{*}{$n \in \mathcal{F}_{2}$} & $\begin{array}{c}\frac{1}{N} \sum_{n=1}^{N} P_{\mathrm{B}}(n) \leq P_{\mathrm{av}} \text { can be satisfied } \\
\text { when } \forall n \in \mathcal{F}_{2}, P_{\mathrm{B}}^{*}(n)=P_{\text {peak }}\end{array}$ & $\begin{array}{c}\rho^{*}(n)=\bar{\rho}^{*} \text {, calculated via one-dimension search, } \\
\qquad \alpha^{*}(n), \text { cf. Lemma } 1, \\
P_{\mathrm{B}}^{*}(n)=P_{\text {peak }}\end{array}$ \\
\hline & Otherwise & $\begin{array}{c}\rho^{*}(n)=\bar{\rho}^{*} \text {, calculated via one-dimension search, } \\
\alpha^{*}(n), \text { cf. Lemma } 1, \\
P_{\mathrm{B}}^{*}(n), \text { cf. Proposition } 4\end{array}$ \\
\hline
\end{tabular}

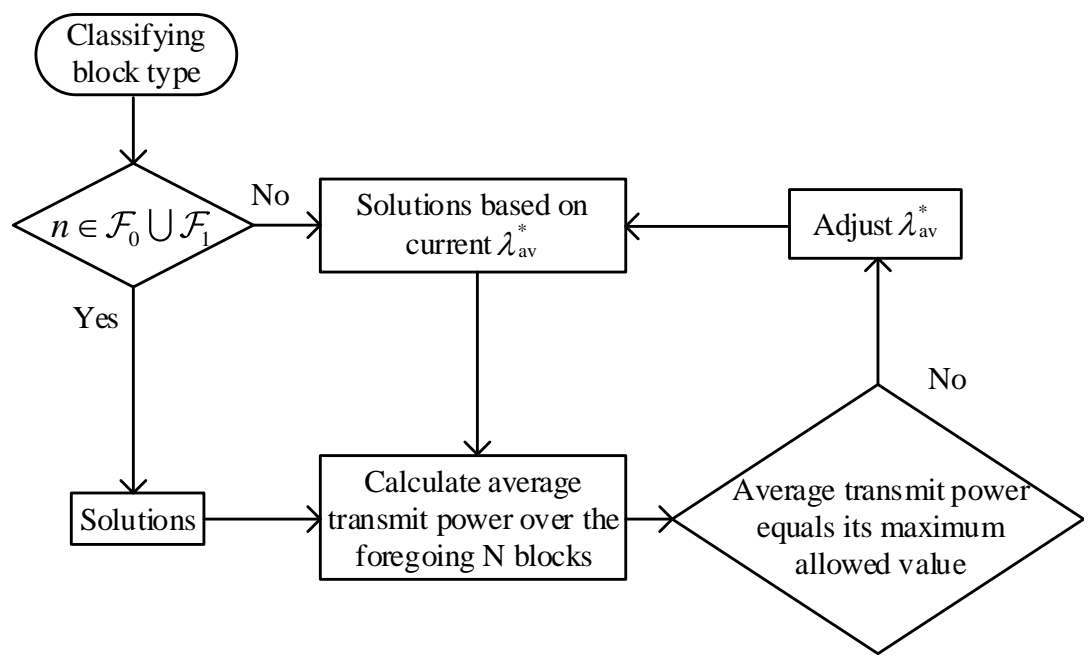

Fig. 2: Procedures to determine the solutions to P1.

\section{Low-Complexity Algorithm}

The key idea of the low-complexity algorithm is that the BS continuously transmits with its maximum allowed average transmit power (i.e., $P_{\mathrm{av}}$ ), and adjusts its power allocation to maximize the interference tolerance at user $\mathrm{N}$, regardless of the possible interference from the BD. On the other hand, for each fading block, the BD first attempts to switch to the fixed 
reflection coefficient (i.e., $\bar{\rho}$ ) to transmit information to user $\mathrm{N}^{11}$. Then, if user $\mathrm{N}$ suffers from $\mathrm{IO}$, it will inform the BD to switch its reflection coefficient to zero so that the performance of the PT will not be impaired. On the contrary, if user $\mathrm{N}$ can recover $x_{\mathrm{N}}$, a transmission rate of $\log _{2}\left(1+\gamma_{\mathrm{NDD}}\right)$ can be achieved during this fading block. This mechanism may introduce additional overheads since user $\mathrm{N}$ has to inform the BD whether the BD needs to switch its reflection coefficient to zero. However, the overheads are negligible since the BD is typically located close to the user.

In summary, for the proposed low-complexity algorithm, the BS only needs to determine the PA factor to maximize the interference tolerance at user $\mathrm{N}$ for each fading block. It follows from (A.1) and (A.5) that this tolerance can be written as $\min \left\{\frac{\left|h_{\mathrm{BN}}\right|^{2} P_{\mathrm{B}}\left(1-\alpha 2^{R_{\mathrm{F}}}\right)}{\left(2^{R_{\mathrm{F}}}-1\right)}-\sigma_{\mathrm{N}}^{2}, \frac{\left|h_{\mathrm{BN}}\right|^{2} P_{\mathrm{B}} \alpha}{\left(2^{R_{\mathrm{N}}}-1\right)}-\sigma_{\mathrm{N}}^{2}\right\}$. Note that we still have to ensure that user $\mathrm{F}$ can recover $x_{\mathrm{F}}$. Therefore, the problem can be formulated as

$$
\mathrm{P} 3: \max _{\alpha} \min \left\{\frac{\left|h_{\mathrm{BN}}\right|^{2} P_{\mathrm{av}}\left(1-\alpha 2^{R_{\mathrm{F}}}\right)}{\left(2^{R_{\mathrm{F}}}-1\right)}-\sigma_{\mathrm{N}}^{2}, \frac{\left|h_{\mathrm{BN}}\right|^{2} P_{\mathrm{av}} \alpha}{\left(2^{R_{\mathrm{N}}}-1\right)}-\sigma_{\mathrm{N}}^{2}\right\},
$$$$
\text { s. t. } 0<\alpha<1 \text { and (9), where } P_{\mathrm{B}}=P_{\mathrm{av}} \text {. }
$$

Proposition 5: When $P_{\mathrm{av}} \leq \frac{\sigma_{\mathrm{F}}^{2}\left(2^{R_{\mathrm{F}}}-1\right)}{\left|h_{\mathrm{BF}}\right|^{2}}$, P3 is infeasible. Otherwise, the optimal PA factor for $\mathrm{P} 3$ is $\alpha^{*}=\min \left\{\frac{1}{2^{R_{\mathrm{F}}}}\left(1-\frac{\sigma_{\mathrm{F}}^{2}\left(2^{R_{\mathrm{F}}}-1\right)}{P_{\mathrm{av}}\left|h_{\mathrm{BF}}\right|^{2}}\right), \frac{2^{R_{\mathrm{N}}-1}}{2^{R_{\mathrm{F}} 2^{R_{\mathrm{N}}}-1}}\right\}$.

Proof: First, we rewrite constraint (9) as $\alpha \leq \frac{1}{2^{R_{\mathrm{F}}}}\left(1-\frac{\sigma_{\mathrm{F}}^{2}\left(2^{R_{\mathrm{F}}}-1\right)}{P_{\mathrm{av}}\left|h_{\mathrm{BF}}\right|^{2}}\right)$. Then, the proof can be readily completed by observing that the first term of the objective function of P3 is a monotonically decreasing function of $\alpha$ and the second term is a monotonically increasing function of $\alpha$.

Note that when P3 is infeasible, or when the optimization result of P3 is a negative value, IO cannot be avoided even if there is no interference from the BD. In this case, the total transmit power at $\mathrm{BS}$ (i.e., $P_{\mathrm{B}}$ ) is set to zero to save energy as before.

So far, we have completed the development of the low-complexity algorithm. As can be observed from Proposition 5, the low-complexity algorithm only involves simple computation. Also, it only requires the CSI of the BS-N link and the BS-F link, which is typically available at the BS for a NOMA downlink transmission system. In other words, the proposed low-complexity

\footnotetext{
${ }^{11}$ Note that the power consumption constraint still needs to be considered. Specifically, for a fading block when (10) cannot be satisfied with the reflection coefficient equal to $\bar{\rho}$, the BD switches its reflection coefficient to zero.
} 
algorithm indeed reduces both the computational complexity and the signaling overheads compared with the algorithm presented in Section III-B.

\section{NumericAl Results AND Discussion}

In this section, representative numerical results are presented to demonstrate and compare the performance of the derived solutions. Specifically, we assume i.i.d. Rayleigh fading for all the involved channels [12], and each result is obtained by averaging over $5 \times 10^{5}$ channel realizations. Note that herein the assumption of the channel fading types does not affect the derived solutions, since the derivation does not rely on the specific distributions or values of channel coefficients. Without loss of generality, the system parameters setup is adopted as in Table II unless otherwise specified. The noise variances $\left(\sigma_{\mathrm{N}}^{2}\right.$ and $\left.\sigma_{\mathrm{F}}^{2}\right)$ are normalized to one, and the ratio of the transmit power to the normalized noise variance in $\mathrm{dB}$ is used to measure the strength of the transmit power in the rest of the paper. To make a fair comparison, the peak transmit power at BS for P2 is set to equal the maximum allowed average transmit power at $\mathrm{BS}$ for $\mathrm{P} 1^{12}$ as in [12], which is reasonable since the optimal total transmit power at BS for P2 is always the peak transmit power.

The performance of the Backscatter-NOMA scheme without parameter optimization [14], which we refer to as the baseline scheme in the rest of the paper, is also shown in this section to demonstrate the achievable performance gain of the derived solutions to P1 as well as the lowcomplexity algorithm for P1. To make a fair comparison, for the baseline scheme, we also assume that the BD can switch between a zero reflection coefficient and a fixed reflection coefficient $\bar{\rho}$ for each fading block, and the BD switches to $\bar{\rho}$ only when its interference will not result in IO for the PT and the power consumption constraint at the BD can be satisfied. Meanwhile, each result of the baseline scheme is obtained by adopting the optimal fixed PA factor and the optimal fixed value of $\bar{\rho}^{13}$.

Fig. 3 demonstrates the outage probability of the PT versus the transmit power level at the BS. From the figure, several observations are drawn as follows: 1) The outage probability generally

\footnotetext{
${ }^{12}$ Note that the peak transmit power constraint is considered in both $\mathrm{P} 1$ and $\mathrm{P} 2$, whereas the average transmit power constraint is considered only in P1.

${ }^{13}$ Remember that our goal is to maximize the ergodic capacity of the ST on the premise of preserving the outage performance of the PT. Therefore, the optimal fixed PA factor is obtained via violent search to minimize the outage probability of the PT, since the outage performance is irrelevant to the choice of $\bar{\rho}$. Then, by adopting the optimal fixed PA factor, the optimal fixed value of $\bar{\rho}$ is obtained through violent search to maximize the ergodic capacity of the ST.
} 
TABLE II: Default system parameters.

\begin{tabular}{c|c|c}
\hline Parameter & Symbol & Value \\
\hline Average channel power gain of the BS-N link & $E\left\{\left|h_{\mathrm{BN}}\right|^{2}\right\}$ & 0.9 \\
\hline Average channel power gain of the BS-F link & $E\left\{\left|h_{\mathrm{BF}}\right|^{2}\right\}$ & 0.3 \\
\hline Average channel power gain of the BS-BD link & $E\left\{\left|h_{\mathrm{BD}}\right|^{2}\right\}$ & 0.9 \\
\hline Average channel power gain of the BD-N link & $E\left\{\left|h_{\mathrm{DN}}\right|^{2}\right\}$ & 0.5 \\
\hline Target rate at user N & $R_{\mathrm{N}}$ & $1 \mathrm{bit} / \mathrm{s} / \mathrm{Hz}$ \\
\hline Target rate at user F & $R_{\mathrm{F}}$ & $0.5 \mathrm{bit} / \mathrm{s} / \mathrm{Hz}$ \\
\hline Energy conversion efficiency at BD & $\eta_{\mathrm{C}}$ & 0.5 \\
\hline Backscatter efficiency at BD & $\eta_{\mathrm{B}}$ & 0.5 \\
\hline Static power consumption at BD & $\epsilon_{s}$ & 0.1 \\
\hline $\begin{array}{c}\text { Dynamic power consumption coefficient at BD } \\
\text { The ratio of the peak transmit power to the } \\
\text { maximum allowed average transmit power }\end{array}$ & $\epsilon_{d}$ & 0.1 \\
\hline$P_{\mathrm{av}}$ & 2 \\
\hline
\end{tabular}

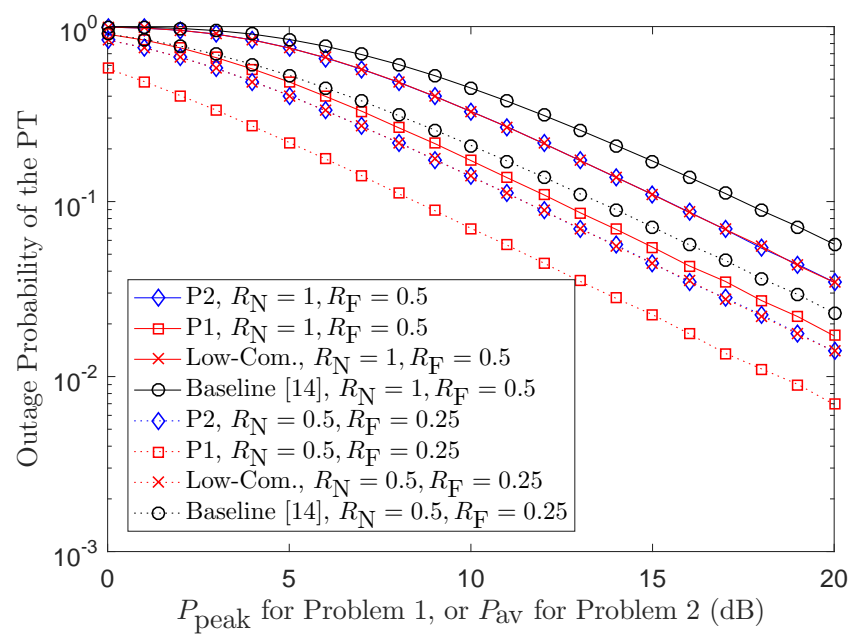

Fig. 3: Effects of the transmit power level on the outage probability.

decreases with the transmit power level at BS, whereas the outage probability for P2 is higher than that for $\mathrm{P} 1$. This is because for both $\mathrm{P} 1$ and $\mathrm{P} 2$, the outage probability can be written as in Corollary 1, which is a monotonically decreasing function of the peak transmit power at BS and is irrelevant to the average transmit power constraint as long as the peak transmit power is larger 
than the maximum allowed average transmit power ${ }^{14}$; 2) The performance of the low-complexity algorithm for P1 is shown to be inferior to that of the developed solutions to P1 in terms of outage probability. This is because for the low-complexity algorithm, the BS always transmits with its maximum allowed average transmit power, in which case the peak transmit power is essentially equal to the average transmit power. Note that this performance gap is determined by the gap between the peak transmit power and the maximum allowed average transmit power; 3 ) Both the low-complexity algorithm for P1 and the developed solutions to P1 are superior to the baseline scheme in terms of the outage probability, which benefits from the adaptive parameter optimization.

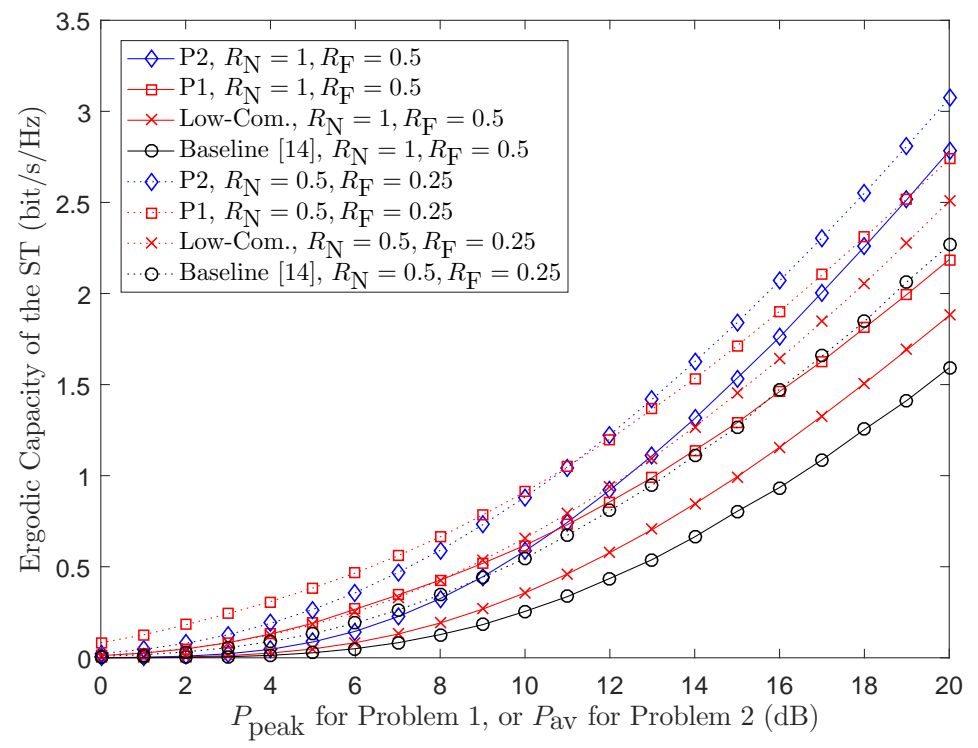

Fig. 4: Effects of the transmit power level on the ergodic capacity.

Fig. 4 shows the ergodic capacity of the ST versus the transmit power level at the BS. From the figure, several observations can be drawn as follows: 1) The ergodic capacity generally increases with the transmit power level at BS, whereas the ergodic capacity for P2 is smaller than that for P1 at first but gradually exceeds P1 with the increase of the transmit power level. This can be explained by the fact that when the power level is low, the optimal reflection coefficient at $\mathrm{BD}$ is either zero or a small value within most fading blocks to harvest enough energy for its

\footnotetext{
${ }^{14}$ Remember that the peak transmit power at BS for P2 is set to equal the maximum allowed average transmit power at BS for P1, and the peak transmit power for P1 is set to double the maximum allowed average transmit power.
} 
circuit operation. In this case, the flexibility from the higher peak transmit power in P1 leads to a higher ergodic capacity. On the contrary, when the power level is high, the optimal reflection coefficient at BD could vary within a wide range due to the dynamic fluctuating characteristics of wireless channels. In this case, the flexibility from the continuously adjustable reflection coefficient in P2 leads to a higher ergodic capacity; 2) When the target data rates of PT are lower, the ergodic capacity of ST is improved. This is due to the fact that when the target data rates of PT are lower, the interference tolerance of PT is improved. In this case, the BD can backscatter a higher power and thus a higher ergodic capacity can be achieved for ST; 3) The performance gap between the low-complexity algorithm for P1 and the developed solutions to P1 is not significant in terms of the ergodic capacity. This demonstrates the effectiveness of the proposed low-complexity algorithm; 4) Both the low-complexity algorithm for P1 and the developed solutions to P1 outperform the baseline scheme in terms of the ergodic capacity, which again shows the importance of adaptive parameter optimization.

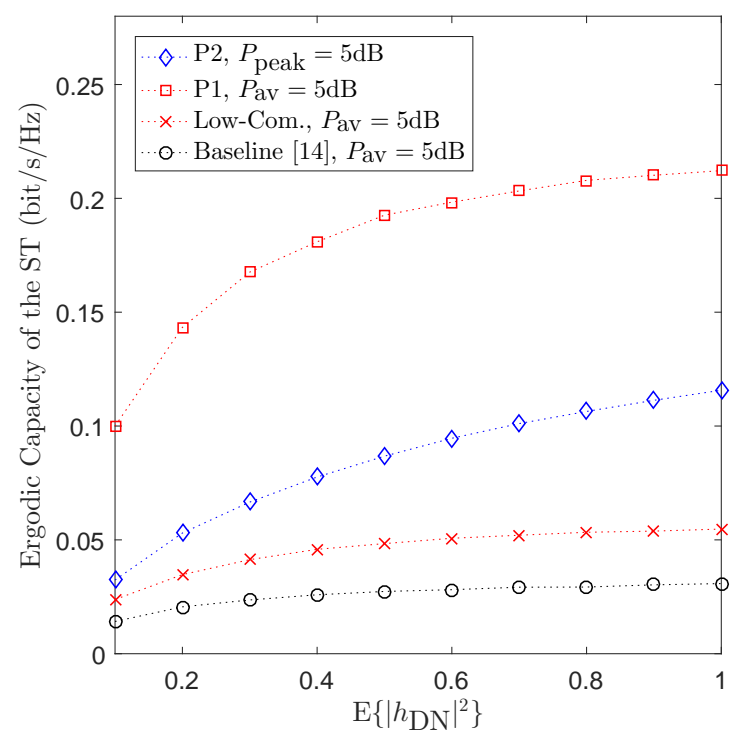

(a)

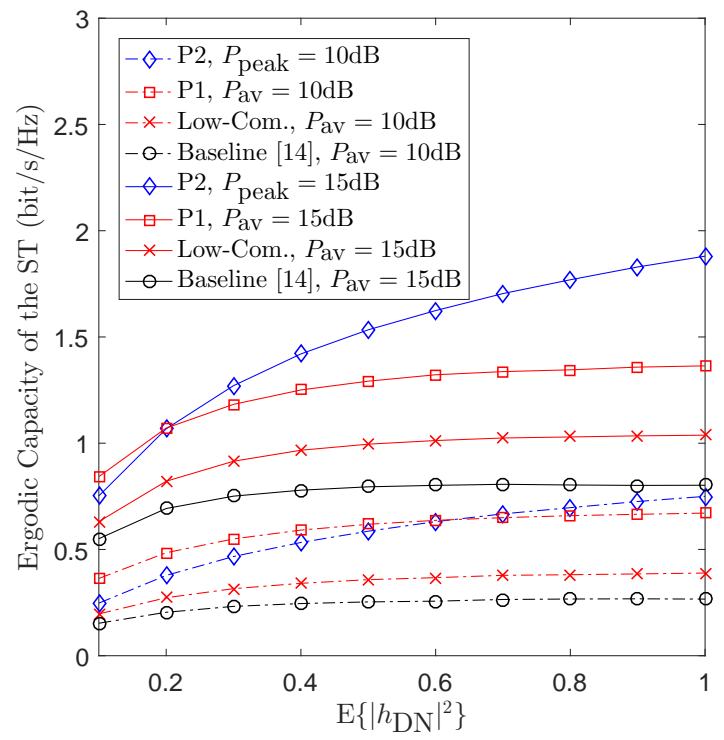

(b)

Fig. 5: Effects of the average channel power gain of the BD-N link on the ergodic capacity.

Fig. 5 shows the effects of the average channel power gain of the BD-N link (i.e., $E\left\{\left|h_{\mathrm{DN}}\right|^{2}\right\}$ ) 
on the ergodic capacity ${ }^{15}$ of the ST. It is intuitive that a higher $E\left\{\left|h_{\mathrm{DN}}\right|^{2}\right\}$ leads to a larger ergodic capacity. An interesting observation is that for the solutions to P1, the low-complexity algorithm for P1, and the baseline scheme, the ergodic capacity first increases and then becomes saturated with the increase of $E\left\{\left|h_{\mathrm{DN}}\right|^{2}\right\}$. This can be explained by the fixed reflection coefficient setup. Specifically, when the backscattered signal is too strong, the BD has to switch its reflection coefficient to zero to avoid impairing the outage performance of the PT, which leads to a zero instantaneous transmission rate of the ST. With the increase of $E\left\{\left|h_{\mathrm{DN}}\right|^{2}\right\}$, this situation happens more frequently and thus the ergodic capacity becomes saturated. In comparison, the reflection coefficient in P2 is continuously adjustable, and thus its reflection coefficient can be tuned to a smaller value, instead of zero, when the channel power gain of the BD-N link is too large.

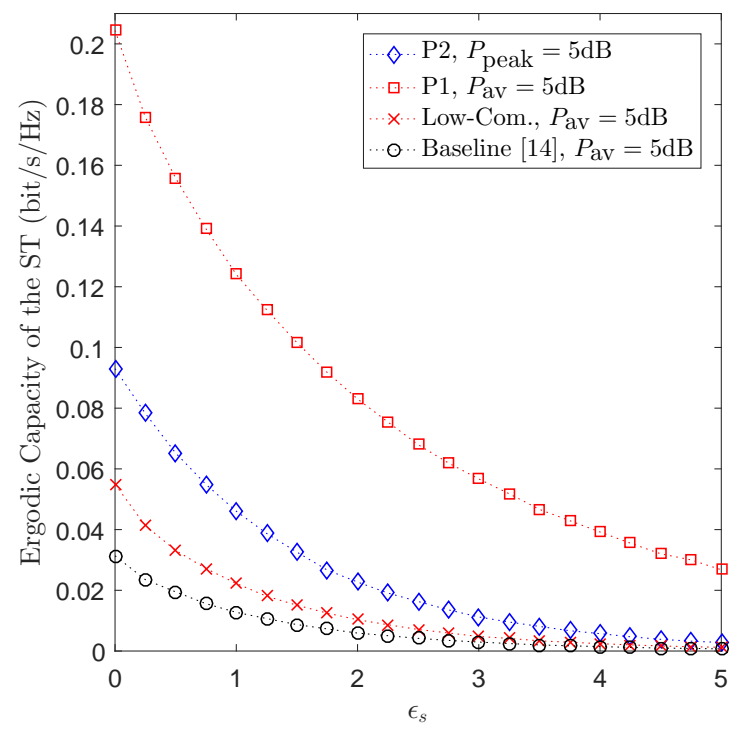

(a)

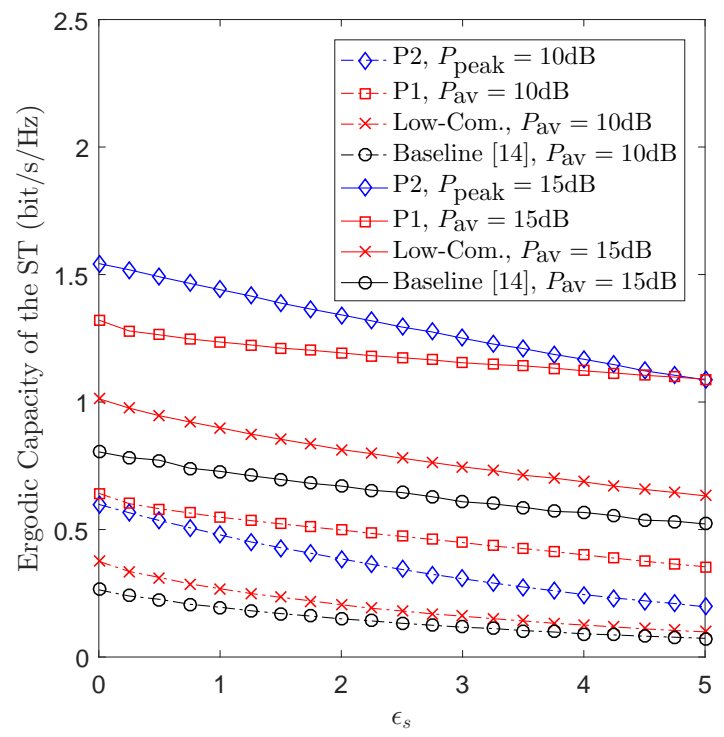

(b)

Fig. 6: Effects of the static power consumption on the ergodic capacity.

Fig. 6 and Fig. 7 demonstrate the effects of the static power consumption and the dynamic power consumption coefficient on the ergodic capacity of the ST, respectively. As can be observed, both a higher static power consumption and a higher dynamic power consumption coefficient may impair the ergodic capacity. However, for a higher transmit power level, the

\footnotetext{
${ }^{15}$ Note that the change of the channel condition of the BD-N link has no effect on the outage probability of PT, as per Corollary 1 . Thus, the corresponding outage probability is not presented herein.
} 


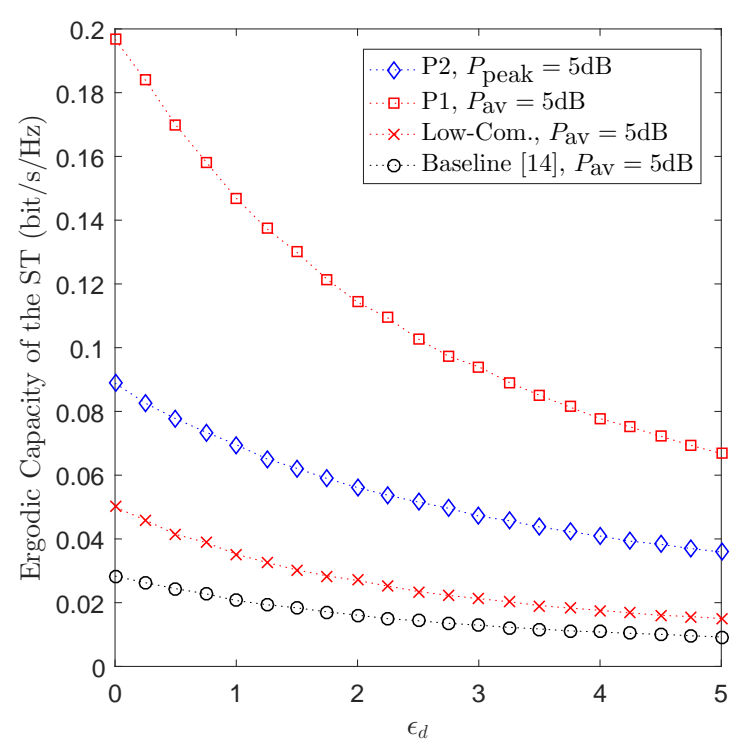

(a)

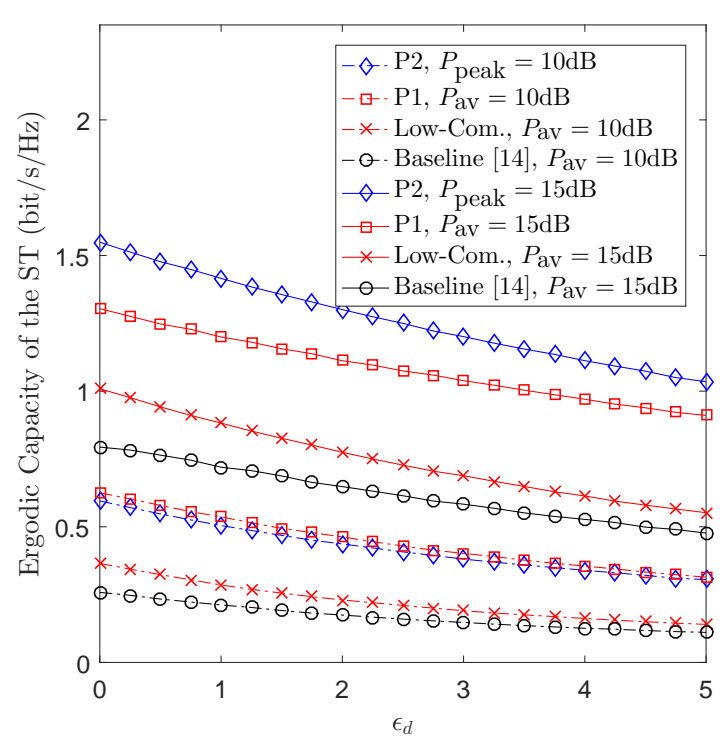

(b)

Fig. 7: Effects of the dynamic power consumption coefficient on the ergodic capacity.

dynamic power consumption coefficient has a larger impact. In comparison, for a lower transmit power level, the static power consumption has a larger impact. This can be explained by the fact that when the transmit power level is high, the transmit rate of the backscatter link is high and thus the ergodic capacity is sensitive to the dynamic power consumption coefficient. On the contrary, when the transmit power level is low, the BD can hardly harvest enough energy to support its basic circuit operation. In this case, the ergodic capacity is sensitive to the static power consumption.

Fig. 8 shows the effects of the peak transmit power on the ergodic capacity of the ST for P1. In the figure, different curves are obtained under different maximum allowed average transmit power. As can be observed, the ergodic capacity first increases and then becomes saturated with the increase of the peak transmit power for each given maximum allowed average transmit power. This can be explained by the fact that although a higher peak transmit power provides a higher flexibility, when the peak transmit power is high enough, the ergodic capacity is mainly restricted by the maximum allowed average transmit power. 


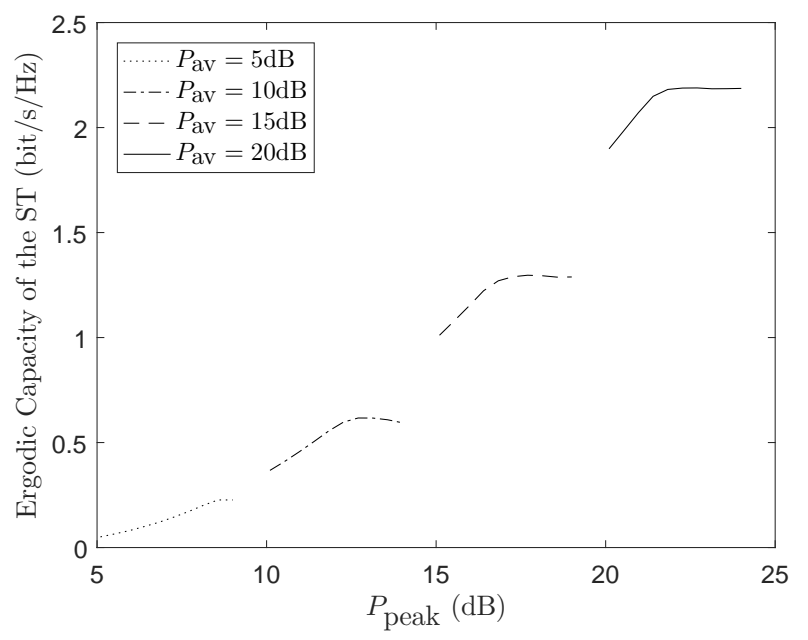

Fig. 8: Effects of the peak transmit power on the ergodic capacity for P1.

\section{CONCLUding REMARKS}

In this paper, we investigated the system parameter optimization problem to maximize the ergodic capacity of the backscatter transmission from the BD to the cell-center user on the premise of preserving the outage performance of NOMA downlink transmission from the BS to the users. We first derived the analytical expressions of the optimal reflection coefficient at $\mathrm{BD}$, the optimal total transmit power at BS, and the optimal power allocation at BS for each fading block under a benchmark scenario with the power consumption constraint at BD as well as the peak transmit power constraint at BS. Furthermore, we took the average transmit power constraint as well as the fixed reflection coefficient constraint into account, and developed an algorithm to determine transmit power setup at BS as well as the choice of the reflection coefficient at BD for each fading block under a given value of the fixed reflection coefficient. We also proposed a low-complexity algorithm to reduce the computational complexity and the signaling overheads. Finally, numerical results showed that the performance loss of the lowcomplexity algorithm is not significant, and it can also achieve performance gain compared with a fixed system parameters setup.

\section{APPENDIX A}

\section{A-1: Proof of Proposition 1}


Constraint (7) can be rewritten as

$$
\frac{\left|h_{\mathrm{BN}}\right|^{2} P_{\mathrm{B}}\left(1-\alpha 2^{R_{\mathrm{F}}}\right)}{\left(2^{R_{\mathrm{F}}}-1\right)}-\sigma_{\mathrm{N}}^{2} \geq \rho \eta_{\mathrm{B}} P_{\mathrm{B}}\left|h_{\mathrm{BD}} h_{\mathrm{DN}}\right|^{2},
$$

which is equivalent to

$$
\begin{gathered}
\alpha<\frac{1}{2^{R_{\mathrm{F}}}}, \\
P_{\mathrm{B}} \geq \frac{\left(2^{R_{\mathrm{F}}}-1\right) \sigma_{\mathrm{N}}^{2}}{\left(1-\alpha 2^{R_{\mathrm{F}}}\right)\left|h_{\mathrm{BN}}\right|^{2}},
\end{gathered}
$$

and

$$
\rho \leq \frac{\frac{\left|h_{\mathrm{BN}}\right|^{2} P_{\mathrm{B}}\left(1-\alpha 2^{R_{\mathrm{F}}}\right)}{\left(2^{R_{\mathrm{F}}}-1\right)}-\sigma_{\mathrm{N}}^{2}}{\eta_{\mathrm{B}} P_{\mathrm{B}}\left|h_{\mathrm{BD}} h_{\mathrm{DN}}\right|^{2}} .
$$

In the same way, constraint (8) can be rewritten as

$$
\frac{\left|h_{\mathrm{BN}}\right|^{2} P_{\mathrm{B}} \alpha}{\left(2^{R_{\mathrm{N}}}-1\right)}-\sigma_{\mathrm{N}}^{2} \geq \rho \eta_{\mathrm{B}} P_{\mathrm{B}}\left|h_{\mathrm{BD}} h_{\mathrm{DN}}\right|^{2},
$$

which is equivalent to

$$
P_{\mathrm{B}} \geq \frac{\left(2^{R_{\mathrm{N}}}-1\right) \sigma_{\mathrm{N}}^{2}}{\alpha\left|h_{\mathrm{BN}}\right|^{2}}
$$

and

$$
\rho \leq \frac{\frac{\left|h_{\mathrm{BN}}\right|^{2} P_{\mathrm{B}} \alpha}{\left(2^{R_{\mathrm{N}}-1}\right)}-\sigma_{\mathrm{N}}^{2}}{\eta_{\mathrm{B}} P_{\mathrm{B}}\left|h_{\mathrm{BD}} h_{\mathrm{DN}}\right|^{2}} .
$$

Similarly, constraint (9) can be rewritten as

$$
P_{\mathrm{B}}\left(1-\alpha 2^{R_{\mathrm{F}}}\right) \geq \frac{\sigma_{\mathrm{F}}^{2}\left(2^{R_{\mathrm{F}}}-1\right)}{\left|h_{\mathrm{BF}}\right|^{2}},
$$

which is equivalent to (A.2) and

$$
P_{\mathrm{B}} \geq \frac{\left(2^{R_{\mathrm{F}}}-1\right) \sigma_{\mathrm{F}}^{2}}{\left(1-\alpha 2^{R_{\mathrm{F}}}\right)\left|h_{\mathrm{BF}}\right|^{2}} .
$$

So far, we have rewritten constraints (7) (9) as constraints (A.2) (A.4), (A.6), (A.7), and (A.9). In the following, we determine the conditions on which these constraints can be satisfied simultaneously (by properly choosing $\rho, \alpha$, and $P_{\mathrm{B}}$ ) when constraint (11) is taken into account.

Note that constraints (A.4) and (A.7) can be neglected herein since they can be readily satisfied by setting $\rho$ to zero. Next, one can show that that constraints (11), (A.2), (A.3), (A.6), and (A.9) 
can be satisfied simultaneously via a proper transmit power setup at BS if and only if there exists an $\alpha \in\left(0,1 / 2^{R_{\mathrm{F}}}\right)$ that makes

$$
P_{\text {peak }} \geq \max \left\{\frac{\left(2^{R_{\mathrm{F}}}-1\right) \sigma_{\mathrm{N}}^{2}}{\left(1-\alpha 2^{R_{\mathrm{F}}}\right)\left|h_{\mathrm{BN}}\right|^{2}}, \frac{\left(2^{R_{\mathrm{N}}}-1\right) \sigma_{\mathrm{N}}^{2}}{\alpha\left|h_{\mathrm{BN}}\right|^{2}}, \frac{\left(2^{R_{\mathrm{F}}}-1\right) \sigma_{\mathrm{F}}^{2}}{\left(1-\alpha 2^{R_{\mathrm{F}}}\right)\left|h_{\mathrm{BF}}\right|^{2}}\right\} .
$$

By observing that both the first term and the third term of the right hand side (RHS) of (A.10) are monotonically increasing functions of $\alpha$, and the second term of the RHS of (A.10) is a monotonically decreasing function of $\alpha$, one can show that when $\frac{\left|h_{\mathrm{BF}}\right|^{2}}{\sigma_{\mathrm{F}}^{2}} \geq \frac{\left|h_{\mathrm{BN}}\right|^{2}}{\sigma_{\mathrm{N}}^{2}}$, the RHS of (A.10)

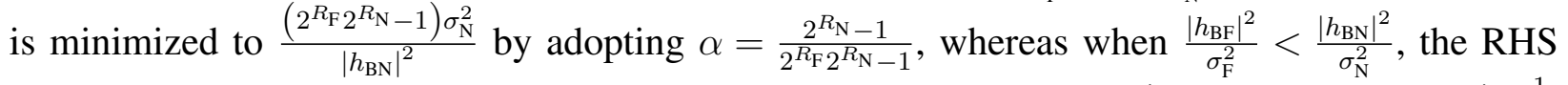
of (A.10) is minimized to $\frac{\left(2^{R_{\mathrm{F}}}-1\right) \sigma_{\mathrm{F}}^{2}}{\left|h_{\mathrm{BF}}\right|^{2}}+\frac{2^{R_{\mathrm{F}}}\left(2^{R_{\mathrm{N}}}-1\right) \sigma_{\mathrm{N}}^{2}}{\left|h_{\mathrm{BN}}\right|^{2}}$ by adopting $\alpha=\left(\frac{\left|h_{\mathrm{BN}}\right|^{2}\left(2^{R_{\mathrm{F}}}-1\right) \sigma_{\mathrm{F}}^{2}}{\left|h_{\mathrm{BF}}\right|^{2}\left(2^{R_{\mathrm{N}}}-1\right) \sigma_{\mathrm{N}}^{2}}+2^{R_{\mathrm{F}}}\right)^{-1}$. Combining these two cases, one can conclude that by adopting

$$
\alpha=\alpha_{\mathrm{LB}} \triangleq \min \left\{\frac{2^{R_{\mathrm{N}}}-1}{2^{R_{\mathrm{F}}} 2^{R_{\mathrm{N}}}-1},\left(\frac{\left|h_{\mathrm{BN}}\right|^{2}\left(2^{R_{\mathrm{F}}}-1\right) \sigma_{\mathrm{F}}^{2}}{\left|h_{\mathrm{BF}}\right|^{2}\left(2^{R_{\mathrm{N}}}-1\right) \sigma_{\mathrm{N}}^{2}}+2^{R_{\mathrm{F}}}\right)^{-1}\right\},
$$

the RHS of (A-10) is minimized to $\max \left\{\frac{\left(2^{\left.R_{\mathrm{F}} 2^{R_{\mathrm{N}}}-1\right) \sigma_{\mathrm{N}}^{2}}\right.}{\left|h_{\mathrm{BN}}\right|^{2}}, \frac{\left(2^{R_{\mathrm{F}}}-1\right) \sigma_{\mathrm{F}}^{2}}{\left|h_{\mathrm{BF}}\right|^{2}}+\frac{2^{R_{\mathrm{F}}}\left(2^{R_{\mathrm{N}}}-1\right) \sigma_{\mathrm{N}}^{2}}{\left|h_{\mathrm{BN}}\right|^{2}}\right\} \triangleq P_{\mathrm{LB}}$, which means that constraints $(7) \sim(9)$ and (11) can be satisfied simultaneously by properly setting the transmit power at BS and the reflection coefficient at BD if and only if

$$
P_{\text {peak }} \geq P_{\mathrm{LB}}
$$

Note that when the reflection coefficient at BD $(\rho)$ equals zero, IO can be avoided by setting $\alpha=\alpha_{\mathrm{LB}}$ and $P_{\mathrm{B}} \geq P_{\mathrm{LB}}$ for a given fading block if and only if (A.12) holds. Therefore, for both P1 and P2, the outage probability of PT can be written as in Corollary 1. In addition, note that when $\rho$ equals zero, the minimum required transmit power at BS to avoid IO for PT is exactly $P_{\mathrm{LB}}$. This conclusion is useful when considering the average transmit power constraint in P1.

Now we turn to investigate when the constraints (10) and (11) can be satisfied simultaneously for P2. Note that the left hand side (LHS) of (10) is a monotonically increasing function of $P_{\mathrm{B}}$ and is also a monotonically decreasing function of $\rho$, whereas the RHS of (10) can be minimize to $\epsilon_{s}$ by setting $\rho=0$. Combining these observations with constraint (11), one can show that for P2, constraints (10) and (11) can be satisfied simultaneously by properly setting the total transmit power at BS and the reflection coefficient at BD if and only if

$$
\eta_{\mathrm{C}} P_{\text {peak }}\left|h_{\mathrm{BD}}\right|^{2} \geq \epsilon_{s}
$$

Finally, when both (A.12) and (A.13) are satisfied, one can easily determine that P2 is feasible by setting $\alpha=\alpha_{\mathrm{LB}}, P_{\mathrm{B}}=P_{\text {peak }}$, and $\rho=0$. This completes the proof. 


\section{A-2: Proof of Proposition 2}

Firstly, given the PA factor and the total transmit power at BS, we consider the optimal reflection coefficient at $\mathrm{BD}$ for a given feasible fading block. By noting that the LHS of (10) is a monotonically decreasing function of $\rho$ and the RHS of (10) is a monotonically increasing function of $\rho$, we can rewrite constraint (10) as

$$
\rho \leq \hat{\rho}
$$

where $\hat{\rho}$ denotes the unique solution of the equation $(1-\rho) \eta_{\mathrm{C}} P_{\mathrm{B}}\left|h_{\mathrm{BD}}\right|^{2}=\epsilon_{s}+\epsilon_{d} \log _{2}\left(1+\gamma_{\mathrm{NDD}}\right)$ and can be readily determined by using the dichotomy algorithm. Note that $\hat{\rho} \in[0,1)$ definitely exists since the inequality $\eta_{\mathrm{C}} P_{\mathrm{B}}\left|h_{\mathrm{BD}}\right|^{2} \geq \epsilon_{s}$ holds for a feasible fading block as per Proposition 1. Next, by observing that the objective function of $\mathrm{P} 2$ is a monotonically increasing function of $\rho$ and combing constraints (A.4), (A.7), and (A.14) (these are all of the constraints related to $\rho)$, we can arrive at (15).

Now we consider the optimal PA factor at BS. Note that the objective function of P2 does not contain $\alpha$ directly but the optimal reflection coefficient $\left(\rho^{*}\right)$ in (15) is relevant to $\alpha$. Meanwhile, the objective function of $\mathrm{P} 2$ is a monotonically increasing function of $\rho$. Therefore, given the total transmit power at BS and with the reflection coefficient equal to $\rho^{*}$, the optimal PA factor $\left(\alpha^{*}\right)$ should maximize the RHS of (15). For such, note that the first term of the RHS of (15) is irrelevant to $\alpha$, the second term of the RHS of (15) is a monotonically increasing function of $\alpha$, and the third term of the RHS of (15) is a monotonically decreasing function of $\alpha$. Therefore, to maximize the RHS of (15), $\alpha^{*}$ should make the second term equal to the third term. Combining this observation with constraints (A.2), (A.3), (A.6), and (A.9) (apart from the constraints related to $\rho$, which have been considered, these are all of the constraints related to $\alpha$ ), we can arrive at (16).

Finally, we determine the optimal total transmit power at BS as follows. Adopting the optimal reflection coefficient in (15) and the optimal PA factor in (16), we have

$$
\rho^{*} P_{\mathrm{B}}=\min \left\{\widehat{\rho} P_{\mathrm{B}}, \frac{\left|h_{\mathrm{BN}}\right|^{2} \frac{\alpha^{*} P_{\mathrm{B}}}{\left(2^{R_{\mathrm{N}}}-1\right)}-\sigma_{\mathrm{N}}^{2}}{\eta_{\mathrm{B}}\left|h_{\mathrm{BD}} h_{\mathrm{DN}}\right|^{2}}, \frac{\left|h_{\mathrm{BN}}\right|^{2} \frac{\left(1-2^{R_{\mathrm{F}}} \alpha^{*}\right) P_{\mathrm{B}}}{\left(2^{R_{\mathrm{F}}}-1\right)}-\sigma_{\mathrm{N}}^{2}}{\eta_{\mathrm{B}}\left|h_{\mathrm{BD}} h_{\mathrm{DN}}\right|^{2}}\right\} .
$$

According to Proposition 2 of [12], the first term of the RHS of (A.15) is a monotonically increasing function of $P_{\mathrm{B}}$. Additionally, note that 1) $\alpha_{\mathrm{U}} P_{\mathrm{B}}$ is a monotonically increasing function of $P_{\mathrm{B}}$, and the second term of the RHS of (A.15) is smaller than the third term when $\alpha^{*}=\alpha_{\mathrm{U}}$; 
2) $\left(1-2^{R_{\mathrm{F}}} \alpha_{\mathrm{L}}\right) P_{\mathrm{B}}$ is a monotonically increasing function of $P_{\mathrm{B}}$, and the third term of the RHS of (A.15) is smaller than the second term when $\left.\alpha^{*}=\alpha_{\mathrm{L}} ; 3\right)$ Both $\hat{\alpha} P_{\mathrm{B}}$ and $\left(1-2^{R_{\mathrm{F}}} \hat{\alpha}\right) P_{\mathrm{B}}$ are monotonically increasing functions of $P_{\mathrm{B}}$. Therefore, we can conclude that the RHS of (A.15) is a monotonically increasing function of $P_{\mathrm{B}}$. On the other hand, so far, all the constraints have been considered except the peak transmit power constraint. Therefore, by observing that the objective function of $\mathrm{P} 2$ is a monotonically increasing function of $\rho P_{\mathrm{B}}$, we have $P_{\mathrm{B}}^{*}=P_{\text {peak }}$, which completes the proof.

\section{ApPENDIX B}

\section{B-1: Proof of Proposition 3}

The sufficient and necessary condition for $n \in \mathcal{F}_{0}$ has been derived in Appendix A-1. In the following, we assume that inequality (A.12) holds (i.e., $n \notin \mathcal{F}_{0}$ ) and determine the conditions for $n \in \mathcal{F}_{1}$ and $n \in \mathcal{F}_{2}$. In other words, in this part, we derive the conditions on which constraints (7) (11) can be satisfied simultaneously (via a proper transmit power setup at BS) with the reflection coefficient equal to $\bar{\rho}$. For conciseness, we omit the block index $n$ hereafter.

Firstly, we rewrite constraints $(7) \sim(10)$ as follows. According to Appendix A-1, constraints (7), (8), and (9) can be rewritten, respectively, as (A.1), (A.5), and (A.8). With the reflection coefficient equal to $\bar{\rho}$ and after some algebraic arrangements, one can further show that (A.1), (A.5), and (A.8) are equivalent to

$$
\begin{gathered}
\frac{\left|h_{\mathrm{BN}}\right|^{2}}{\left(2^{R_{\mathrm{F}}} 2^{R_{\mathrm{N}}}-1\right)}>\bar{\rho} \eta_{\mathrm{B}}\left|h_{\mathrm{BD}} h_{\mathrm{DN}}\right|^{2}, \\
\frac{\bar{\rho} \eta_{\mathrm{B}}\left|h_{\mathrm{BD}} h_{\mathrm{DN}}\right|^{2}\left(2^{R_{\mathrm{N}}}-1\right)}{\left|h_{\mathrm{BN}}\right|^{2}}<\alpha<\frac{1}{2^{R_{\mathrm{F}}}}\left(1-\frac{\bar{\rho} \eta_{\mathrm{B}}\left|h_{\mathrm{BD}} h_{\mathrm{DN}}\right|^{2}\left(2^{R_{\mathrm{F}}}-1\right)}{\left|h_{\mathrm{BN}}\right|^{2}}\right),
\end{gathered}
$$

and

$$
P_{\mathrm{B}} \geq \max \left\{\frac{\sigma_{\mathrm{N}}^{2}}{\frac{\left|h_{\mathrm{BN}}\right|^{2}\left(1-\alpha 2^{R_{\mathrm{F}}}\right)}{\left(2^{R_{\mathrm{F}}}-1\right)}-\bar{\rho} \eta_{\mathrm{B}}\left|h_{\mathrm{BD}} h_{\mathrm{DN}}\right|^{2}}, \frac{\sigma_{\mathrm{N}}^{2}}{\frac{\left|h_{\mathrm{BN}}\right|^{2} \alpha}{\left(2^{R_{\mathrm{N}}-1}\right)}-\bar{\rho} \eta_{\mathrm{B}}\left|h_{\mathrm{BD}} h_{\mathrm{DN}}\right|^{2}}, \frac{\sigma_{\mathrm{F}}^{2}}{\frac{\left|h_{\mathrm{BF}}\right|^{2}\left(1-\alpha 2^{R_{\mathrm{F}}}\right)}{\left(2^{R_{\mathrm{F}}}-1\right)}}\right\} .
$$

Note that when (B.1) holds, (B.2) is not an empty set. On the other hand, constraint (10) can be rewritten as

$$
P_{\mathrm{B}} \geq \widehat{P_{\mathrm{B}}}
$$


where $\widehat{P_{\mathrm{B}}}$ denotes the unique solution of the equation $(1-\bar{\rho}) \eta_{\mathrm{C}} P_{\mathrm{B}}\left|h_{\mathrm{BD}}\right|^{2}=\epsilon_{s}+\epsilon_{d} \log _{2}\left(1+\bar{\gamma}_{\mathrm{NDD}}\right)$ and can be readily determined by using the dichotomy algorithm.

Next, provided that (B.1) holds, one can show that constraints (B.2), (B.3), (B.4), and (11) can be satisfied simultaneously (via a proper transmit power setup at BS) if and only if there exists an $\alpha$ satisfying (B.2) that makes

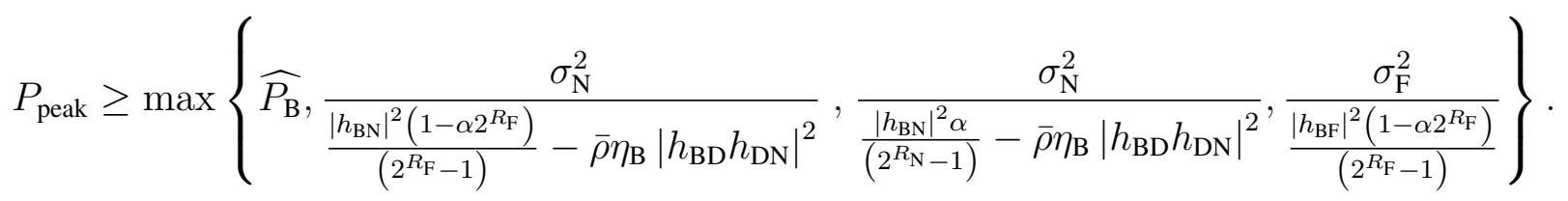

Note that the first term of the RHS of (B.5) is irrelevant to $\alpha$.

Now we concentrate on the second, third, and fourth terms of the RHS of (B.5). By observing that both the second and the fourth terms are monotonically increasing functions of $\alpha$, whereas the third term is a monotonically decreasing function of $\alpha$, one can show that when $\sigma_{\mathrm{F}}^{2} \bar{\rho} \eta_{\mathrm{B}}\left|h_{\mathrm{BD}} h_{\mathrm{DN}}\right|^{2} \geq \frac{\sigma_{\mathrm{F}}^{2}\left|h_{\mathrm{BN}}\right|^{2}-\sigma_{\mathrm{N}}^{2}\left|h_{\mathrm{BF}}\right|^{2}}{2^{R_{\mathrm{F}} 2^{R_{\mathrm{N}}-1}}}$, the maximum value of the three terms is minimized to

$$
P_{\mathrm{LB} 1} \triangleq \frac{\sigma_{\mathrm{N}}^{2}}{\frac{\left|h_{\mathrm{BN}}\right|^{2}}{2^{R_{\mathrm{F} 2}{ }^{R}-1}}-\bar{\rho} \eta_{\mathrm{B}}\left|h_{\mathrm{BD}} h_{\mathrm{DN}}\right|^{2}}
$$

by adopting

$$
\alpha=\frac{2^{R_{\mathrm{N}}}-1}{2^{R_{\mathrm{F}}} 2^{R_{\mathrm{N}}}-1}
$$

which can be proved to satisfy (B.2) by using inequality (B.1). Otherwise, the maximum value of the three terms is minimized to

$$
P_{\mathrm{LB} 2} \triangleq \frac{\sigma_{\mathrm{N}}^{2}+\frac{\left|h_{\mathrm{BN}}\right|^{2}\left(2^{R_{\mathrm{F}}}-1\right) \sigma_{\mathrm{F}}^{2}}{\left|h_{\mathrm{BF}}\right|^{2}\left(2^{R_{\mathrm{N}}}-1\right) 2^{R_{\mathrm{F}}}}}{\frac{\left|h_{\mathrm{BN}}\right|^{2}}{2^{R_{\mathrm{F}}}\left(2^{R_{\mathrm{N}}}-1\right)}-\bar{\rho} \eta_{\mathrm{B}}\left|h_{\mathrm{BD}} h_{\mathrm{DN}}\right|^{2}}
$$

by adopting

$$
\alpha=\frac{\frac{\sigma_{\mathrm{N}}^{2}\left|h_{\mathrm{BF}}\right|^{2}}{\left(2^{R_{\mathrm{F}}}-1\right)}+\sigma_{\mathrm{F}}^{2} \bar{\rho} \eta_{\mathrm{B}}\left|h_{\mathrm{BD}} h_{\mathrm{DN}}\right|^{2}}{\frac{\sigma_{\mathrm{F}}^{2}\left|h_{\mathrm{BN}}\right|^{2}}{\left(2^{R_{\mathrm{N}}-1}\right)}+\frac{\sigma_{\mathrm{N}}^{2}\left|h_{\mathrm{BF}}\right|^{2} 2^{R_{\mathrm{F}}}}{\left(2^{R_{\mathrm{F}}-1}\right)}}
$$

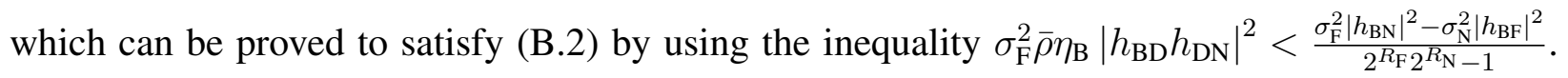

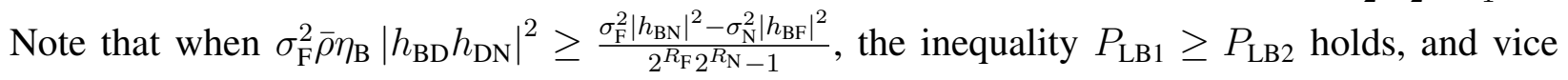
versa. Therefore, summarizing the foregoing results, we can conclude that if and only if (B.1) and $P_{\text {peak }} \geq P_{\mathrm{M}} \triangleq \max \left\{\widehat{P_{\mathrm{B}}}, P_{\mathrm{LB} 1}, P_{\mathrm{LB} 2}\right\}$ hold, constraints $(7) \sim(11)$ can be satisfied simultaneously 
(by setting $\alpha$ according to (B.7) as well as (B.9), and setting $P_{\mathrm{B}} \geq P_{\mathrm{M}}$ ), which completes the proof.

\section{B-2: Proof of Lemma 1}

For conciseness, we omit the block index $n$ herein. Note that constraints (10), (11), and (12) are irrelevant to $\alpha$, and it follows from Appendix B-1 that the remaining constraints (7) (9) are equivalent to constraints $\left(\right.$ B.1) (B.3), among which (B.1) is already satisfied since $n \in \mathcal{F}_{2}$, (B.2) restricts the value of $\alpha$, whereas (B.3) restricts the minimum allowed value of $P_{\mathrm{B}}$ and is relevant to $\alpha$. On the other hand, the objective function in P1 does not contain $\alpha$ directly and is a monotonically increasing function of $P_{\mathrm{B}}$. Based on these two observations, we can conclude that the optimal PA factor at BS $\left(\alpha^{*}\right)$ should minimize the RHS of (B.3) on the premise of satisfying (B.2), which has been given by (B.7) and (B.9). This completes the proof.

\section{B-3: Proof of Proposition 4}

Obviously, for $n \in \mathcal{F}_{2}$, if $P_{\mathrm{M}}(n)=P_{\text {peak }}$, to satisfy the constraints of $\mathrm{P} 1 \mathrm{~b}$, the optimal total transmit power at $\mathrm{BS}$ has to be $P_{\mathrm{B}}^{*}(n)=P_{\text {peak }}$. Therefore, in the following, we only need to determine the optimal total transmit power for the fading blocks in which $P_{\mathrm{M}}(n) \neq P_{\text {peak }}$, and we denote the set of the indexes of such fading blocks as $\mathcal{F}_{2}^{\prime}\left(\mathcal{F}_{2}^{\prime} \subseteq \mathcal{F}_{2}\right)$ such that P1b can be rewritten as

$$
\begin{aligned}
\text { P1c : } \max _{P_{\mathrm{B}}(n), n \in \mathcal{F}_{2}^{\prime}} \frac{1}{N} \sum_{\substack{n=1 \\
n \in \mathcal{F}_{2}^{\prime}}}^{N} \log _{2}\left(1+f(n) P_{\mathrm{B}}(n)\right), \\
\text { s. t. } P_{\mathrm{M}}(n) \leq P_{\mathrm{B}}(n) \leq P_{\text {peak }}, n \in \mathcal{F}_{2}^{\prime}, \\
\frac{1}{N} \sum_{n=1}^{N} P_{\mathrm{B}}(n) \leq P_{\mathrm{av}},
\end{aligned}
$$

where $f(n) \triangleq \frac{\bar{\rho} \eta_{\mathrm{B}}\left|h_{\mathrm{BD}}(n) h_{\mathrm{DN}}(n)\right|^{2}}{\sigma_{\mathrm{N}}^{2}}$. Next, the Lagrange function of P1c can be given by [19]

$$
\begin{aligned}
L= & \frac{1}{N} \sum_{\substack{n=1 \\
n \in \mathcal{F}_{2}^{\prime}}}^{N} \log _{2}\left(1+f(n) P_{\mathrm{B}}(n)\right)+\sum_{\substack{n=1 \\
n \in \mathcal{F}_{2}^{\prime}}}^{N} \lambda_{n}\left(P_{\mathrm{B}}(n)-P_{\mathrm{M}}(n)\right) \\
& +\sum_{\substack{n=1 \\
n \in \mathcal{F}_{2}^{\prime}}}^{N} \lambda_{\text {peak }, n}\left(P_{\text {peak }}-P_{\mathrm{B}}(n)\right)+\lambda_{\mathrm{av}}\left(P_{\mathrm{av}}-\frac{1}{N} \sum_{n=1}^{N} P_{\mathrm{B}}(n)\right)
\end{aligned}
$$


where $\lambda_{n}, \lambda_{\text {peak,n}}$, and $\lambda_{\text {av }}$ are dual variables. The corresponding Karush-Kuhn-Tucker (KKT) conditions are

$$
\begin{gathered}
\lambda_{n}^{*}, \lambda_{\text {peak }, n}^{*}, \lambda_{\text {av }}^{*} \geq 0, n \in \mathcal{F}_{2}^{\prime}, \\
P_{\mathrm{B}}^{*}(n) \geq P_{\mathrm{M}}(n), n \in \mathcal{F}_{2}^{\prime}, \\
P_{\mathrm{B}}^{*}(n) \leq P_{\text {peak }}, n \in \mathcal{F}_{2}^{\prime}, \\
\frac{1}{N} \sum_{n=1}^{N} P_{\mathrm{B}}^{*}(n) \leq P_{\text {av }}, \\
\lambda_{n}^{*}\left(P_{\mathrm{B}}^{*}(n)-P_{\mathrm{M}}(n)\right)=0, n \in \mathcal{F}_{2}^{\prime}, \\
\lambda_{\text {peak }, n}^{*}\left(P_{\text {peak }}-P_{\mathrm{B}}^{*}(n)\right)=0, n \in \mathcal{F}_{2}^{\prime}, \\
\lambda_{\text {av }}^{*}\left(P_{\text {av }}-\frac{1}{N} \sum_{n=1}^{N} P_{\mathrm{B}}^{*}(n)\right)=0, \\
\frac{1}{N} \frac{1}{\ln 2\left(1+f(n) P_{\mathrm{B}}^{*}(n)\right)}+\lambda_{n}^{*}-\lambda_{\text {peak }, n}^{*}-\frac{1}{N} \lambda_{\text {av }}^{*}=0, n \in \mathcal{F}_{2}^{\prime} .
\end{gathered}
$$

To solve the KKT conditions listed above, we first prove that the optimal transmit power at BS satisfies

$$
P_{\mathrm{av}}=\frac{1}{N} \sum_{n=1}^{N} P_{\mathrm{B}}^{*}(n)
$$

by contradiction: Assume that $P_{\mathrm{av}} \neq \frac{1}{N} \sum_{n=1}^{N} P_{\mathrm{B}}^{*}(n)$, then it follows from (B.18) that $\lambda_{\mathrm{av}}^{*}=$ 0 . On the other hand, since there exists an $\hat{n} \in \mathcal{F}_{2}^{\prime}$ subject to $P_{\mathrm{B}}^{*}(\hat{n})<P_{\text {peak }}{ }^{16}$, it follows from (B.17) that $\lambda_{\text {peak }, \hat{n}}^{*}=0$. Next, substituting $\lambda_{\text {av }}^{*}=0$ and $\lambda_{\text {peak }, \hat{n}}^{*}=0$ into (B.19), we have $\frac{1}{N} \frac{f(\hat{n})}{\ln 2\left(1+f(\hat{n}) P_{\mathrm{B}}^{*}(\hat{n})\right)}+\lambda_{\hat{n}}^{*}=0$, which contradicts (B.12) since the term $\frac{1}{N} \frac{f(\hat{n})}{\ln 2\left(1+f(\hat{n}) P_{\mathbf{B}}^{*}(\hat{n})\right)}$ is definitely larger than zero. This completes the proof of (B.20).

Next, for a given $n \in \mathcal{F}_{2}^{\prime}$, if the inequalities $\lambda_{n}^{*}>0$ and $\lambda_{\text {peak }, n}^{*}>0$ hold simultaneously, it follows from (B.16) and (B.17) that $P_{\mathrm{M}}(n)=P_{\text {peak, }}$, which contradicts our definition of the set

\footnotetext{
${ }^{16} \mathrm{~A}$ precondition of Proposition 4 is that there exists at least an $\hat{n} \in \mathcal{F}_{2}$ subject to $P_{\mathrm{B}}^{*}(\hat{n})<P_{\text {peak }}$ (otherwise, $\forall n \in \mathcal{F}_{2}$, $\left.P_{\mathrm{B}}^{*}(n)=P_{\text {peak }}\right)$. Note that $\hat{n}$ definitely belongs to $\mathcal{F}_{2}^{\prime}$ as per the definition of the set $\mathcal{F}_{2}^{\prime}$.
} 
$\mathcal{F}_{2}^{\prime}$. As a result, for each $n \in \mathcal{F}_{2}^{\prime}$, there are three cases:

Case 1: $\lambda_{n}^{*}=0, \lambda_{\text {peak }, n}^{*}=0$

In this case, it follows from (B.19) that

$$
P_{\mathrm{B}}^{*}(n)=\frac{1}{\ln 2 \lambda_{\mathrm{av}}^{*}}-\frac{1}{f(n)} .
$$

Next, according to (B.13) and (B.14), we can determine that (B.21) holds when $\frac{f(n)}{\ln 2\left(1+f(n) P_{\text {peak }}\right)} \leq$ $\lambda_{\mathrm{av}}^{*} \leq \frac{f(n)}{\ln 2\left(1+f(n) P_{\mathrm{M}}(n)\right)}$.

Case 2: $\lambda_{n}^{*}>0, \lambda_{\text {peak }, n}^{*}=0$

In this case, it follows from (B.16) that

$$
P_{\mathrm{B}}^{*}(n)=P_{\mathrm{M}}(n)
$$

Next, according to (B.19), we have $\lambda_{n}^{*}=\frac{1}{N}\left(\lambda_{\mathrm{av}}^{*}-\frac{f(n)}{\ln 2\left(1+f(n) P_{\mathrm{M}}(n)\right)}\right)$. Since $\lambda_{n}^{*}>0$ in Case 2, we can determine that (B.22) holds when $\lambda_{\mathrm{av}}^{*}>\frac{f(n)}{\ln 2\left(1+f(n) P_{\mathrm{M}}(n)\right)}$.

Case $3: \lambda_{n}^{*}=0, \lambda_{\text {peak }, n}^{*}>0$

In this case, it follows from (B.17) that

$$
P_{\mathrm{B}}^{*}(n)=P_{\text {peak }}
$$

Next, according to (B.19), we have $\lambda_{\text {peak }, n}^{*}=\frac{1}{N}\left(\frac{f(n)}{\ln 2\left(1+f(n) P_{\text {peak }}\right)}-\lambda_{\text {av }}^{*}\right)$. Since $\lambda_{\text {peak }, n}^{*}>0$ in Case 3, we can determine that (B.23) holds when $\lambda_{\text {av }}^{*}<\frac{f(n)}{\ln 2\left(1+f(n) P_{\text {peak }}\right)}$. This completes the proof.

\section{REFERENCES}

[1] Z. Ding, X. Lei, G. K. Karagiannidis, R. Schober, J. Yuan, and V. K. Bhargava, "A survey on non-orthogonal multiple access for $5 \mathrm{~g}$ networks: Research challenges and future trends," IEEE J. Select. Areas Commun., vol. 35, no. 10, pp. 2181-2195, Oct. 2017.

[2] L. Dai, B. Wang, Z. Ding, Z. Wang, S. Chen, and L. Hanzo, “A survey of non-orthogonal multiple access for 5g," IEEE Commun. Surveys Tuts., vol. 20, no. 3, pp. 2294-2323, thirdquarter 2018.

[3] S. M. R. Islam, N. Avazov, O. A. Dobre, and K. Kwak, "Power-domain non-orthogonal multiple access (NOMA) in 5G systems: Potentials and challenges," IEEE Commun. Surveys Tuts., vol. 19, no. 2, pp. 721-742, Secondquarter 2017.

[4] Y. Saito, Y. Kishiyama, A. Benjebbour, T. Nakamura, A. Li, and K. Higuchi, "Non-orthogonal multiple access (NOMA) for cellular future radio access," in IEEE VTC Spring, Jun. 2013, pp. 1-5.

[5] A. Benjebbour, Y. Saito, Y. Kishiyama, A. Li, A. Harada, and T. Nakamura, "Concept and practical considerations of non-orthogonal multiple access (NOMA) for future radio access," in IEEE ISPACS, Nov. 2013, pp. 770-774.

[6] N. Zhang, J. Wang, G. Kang, and Y. Liu, "Uplink nonorthogonal multiple access in 5g systems," IEEE Commun. Lett., vol. 20, no. 3, pp. 458-461, Mar. 2016.

[7] H. Sadia, M. Zeeshan, and S. A. Sheikh, "Performance analysis of downlink power domain NOMA under fading channels," in ELEKTRO, May 2018, pp. 1-6. 
[8] Z. Ding, M. Peng, and H. V. Poor, "Cooperative non-orthogonal multiple access in 5G systems," IEEE Commun. Lett., vol. 19, no. 8, pp. 1462-1465, Aug. 2015.

[9] V. Liu, A. Parks, V. Talla, S. Gollakota, D. Wetherall, and J. Smith, "Ambient backscatter: Wireless communication out of thin air," in Proc. ACM SIGCOMM, Sep. 2013, pp. 39-50.

[10] N. Van Huynh, D. T. Hoang, X. Lu, D. Niyato, P. Wang, and D. I. Kim, "Ambient backscatter communications: A contemporary survey," IEEE Commun. Surveys Tuts., vol. 20, no. 4, pp. 2889-2922, Fourthquarter 2018.

[11] G. Yang, Q. Zhang, and Y. Liang, "Cooperative ambient backscatter communications for green internet-of-things," IEEE Internet Things J., vol. 5, no. 2, pp. 1116-1130, Apr. 2018.

[12] X. Kang, Y. Liang, and J. Yang, "Riding on the primary: A new spectrum sharing paradigm for wireless-powered IoT devices," IEEE Trans. Wireless Commun., vol. 17, no. 9, pp. 6335-6347, Sep. 2018.

[13] H. Guo, Y. Liang, R. Long, and Q. Zhang, “Cooperative ambient backscatter system: A symbiotic radio paradigm for passive IoT," IEEE Wireless Commun. Lett., vol. 8, no. 4, pp. 1191-1194, Aug. 2019.

[14] Q. Zhang, L. Zhang, Y. Liang, and P. Kam, "Backscatter-NOMA: A symbiotic system of cellular and internet-of-things networks," IEEE Access, vol. 7, pp. 20000-20013, Feb. 2019.

[15] W. Wang, S. He, L. Sun, T. Jiang, and Q. Zhang, "Cross-technology communications for heterogeneous IoT devices through artificial doppler shifts," IEEE Transactions on Wireless Communications, vol. 18, no. 2, pp. 796-806, Feb. 2019.

[16] W. Wang, Y. Chen, L. Wang, and Q. Zhang, "Sampleless Wi-Fi: Bringing low power to Wi-Fi communications," IEEE Trans. Netw., vol. 25, no. 3, pp. 1663-1672, Jun. 2017.

[17] C. Zhong, H. A. Suraweera, G. Zheng, I. Krikidis, and Z. Zhang, "Wireless information and power transfer with full duplex relaying," IEEE Trans. Commun., vol. 62, no. 10, pp. 3447-3461, Oct. 2014.

[18] H. Guo, R. Long, and Y. Liang, “Cognitive backscatter network: A spectrum sharing paradigm for passive IoT,” IEEE Wireless Commun. Lett., vol. 8, no. 5, pp. 1423-1426, Oct. 2019.

[19] S. Boyd and L. Vandenberghe, Convex optimization. Cambridge University Press, 2004. 